\title{
Small-radius jets to all orders in QCD
}

\author{
Mrinal Dasgupta, ${ }^{a}$ Frédéric Dreyer, ${ }^{b, c}$ Gavin P. Salam, ${ }^{d, 1}$ and Gregory Soyez ${ }^{e}$ \\ ${ }^{a}$ Consortium for Fundamental Physics, School of Physics $\&$ Astronomy, University of Manchester, \\ Manchester M13 9PL, United Kingdom \\ ${ }^{b}$ Sorbonne Universités, UPMC Univ Paris 06, UMR 7589, LPTHE, F-75005, \\ Paris, France \\ ${ }^{c}$ CNRS, UMR 7589, LPTHE, F-75005, \\ Paris, France \\ ${ }^{d} \mathrm{CERN}, \mathrm{PH}-\mathrm{TH}$, \\ CH-1211 Geneva 23, Switzerland \\ e IPhT, CEA Saclay, CNRS URA 2306, \\ F-91191 Gif-sur-Yvette, France \\ E-mail: mrinal.dasgupta@manchester.ac.uk, dreyer@lpthe.jussieu.fr, \\ gavin.salam@cern.ch, gregory.soyez@cea.fr
}

AbStract: As hadron collider physics continues to push the boundaries of precision, it becomes increasingly important to have methods for predicting properties of jets across a broad range of jet radius values $R$, and in particular for small $R$. In this paper we resum all leading logarithmic terms, $\alpha_{s}^{n} \ln ^{n} R^{2}$, in the limit of small $R$, for a wide variety of observables. These include the inclusive jet spectrum, jet vetoes for Higgs physics and jet substructure tools. Some of the quantities that we consider are relevant also for heavyion collisions. Furthermore, we examine and comment on the underlying order-by-order convergence of the perturbative series for different $R$ values. Our results indicate that small- $R$ effects can be substantial. Phenomenological studies will appear in a forthcoming companion paper.

KeYwords: QCD Phenomenology, Jets

ArXiv EPRINT: 1411.5182

\footnotetext{
${ }^{1}$ On leave from CNRS, UMR 7589, LPTHE, F-75005, Paris, France
} 


\section{Contents}

1 Introduction 1

2 Jet definitions 3

3 All-order leading-logarithmic resummation 3

3.1 Generating functional evolution equation 5

$\begin{array}{lll}3.2 & \text { Fixed-order expansions for the generating functionals } & 7\end{array}$

$\begin{array}{lll}3.3 & \text { All-order reformulation } & 7\end{array}$

$\begin{array}{llr}4 & \text { Results } & 8\end{array}$

$\begin{array}{llr}4.1 & \text { Inclusive microjet observables } & 9\end{array}$

$\begin{array}{ll}4.2 \text { Hardest microjet observables } & 12\end{array}$

$\begin{array}{lll}\text { 4.2.1 Hardest microjet }\langle\Delta z\rangle & 13\end{array}$

$\begin{array}{lll}4.2 .2 & \text { Jet-veto resummations and }\langle\ln z\rangle & 14\end{array}$

$\begin{array}{ll}4.2 .3 \text { Jet flavour } & 17\end{array}$

$\begin{array}{lll}4.3 & \text { Multi (sub)jet observables } & 19\end{array}$

$\begin{array}{lll}4.3 .1 & \text { Filtering } & 19\end{array}$

$\begin{array}{lll}4.3 .2 & \text { Trimming } & 20\end{array}$

5 Conclusions $\quad 22$

A Analytical expressions $\quad 23$

A.1 Hardest microjet $\langle\Delta z\rangle \quad 23$

A.2 Logarithmic moment $\langle\ln z\rangle \quad 24$

A.3 Jet flavour 26

$\begin{array}{lll}\text { A.4 Filtering } & 26\end{array}$

$\begin{array}{lll}\text { A.5 Trimming } & 27\end{array}$

B Comparisons and fixed-order cross checks $\quad 29$

$\begin{array}{ll}\text { B.1 Comparison with the literature } & 29\end{array}$

$\begin{array}{lll}\text { B.2 Fixed-order cross checks } & 29\end{array}$

\section{Introduction}

Jets, collimated bunches of energetic hadrons, are widely used at hadron colliders and lepton colliders as a proxy for hard quarks or gluons. Procedures to identify jets group particles into clusters, often based on their proximity in angle. Nearly all of the jet algorithms in common use at hadron colliders follow the example of the seminal work by Sterman and Weinberg [1] and involve a parameter that determines how close in angle two 
particles have to be in order to be clustered into the same jet. That parameter is usually referred to as the jet radius, denoted $R$.

From the point of view of perturbative quantum chromodynamics (QCD), it is natural to choose a jet radius of order 1 (see e.g. ref. [2]). However in most practical uses of jets at hadron colliders, the jet radius is taken somewhat smaller: this makes it more straightforward to resolve multiple jets in events such as top-antitop production, which can decay to six quarks and in cascade decays of supersymmetric particles; it also significantly reduces the contamination of the jet by the underlying event and multiple simultaneous $p p$ collisions (pileup). The most common choices for $R$ are in the range $0.4-0.5[3,4]$, and in some extreme environments, such as heavy-ion collisions, even smaller values are used, down to $R=0.2$ [5-9]. Also, one sometimes studies the ratio of the inclusive jet cross-sections obtained with two different $R$ values [5, 10-13]. Additionally, a number of modern jet tools, such as filtering [14] and trimming [15], resolve small subjets within a single moderate- $R$ jet. Yet others build large- $R$ jets from small- $R$ jets [16]. Many of these techniques are described in the reviews [17-20].

A problem with small- $R$ jets, which we generically call "microjets", is that the correspondence between the jet momentum and the original parton's momentum is strongly affected by radiation at angles larger than $R$. This can degrade momentum measurements with the jets, for example, in resonance reconstruction. Furthermore it also affects calculations in perturbative QCD, because the difference between the parton and jet momenta involves an expansion whose dominant terms are $\alpha_{s}^{n} \ln ^{n} R^{2}$, where $\alpha_{s}$ is the strong coupling constant: if $\ln R^{2}$ is sufficiently large, then the series may no longer converge, or do so only very slowly. In such cases in QCD, it is standard to carry out an all-order resummation. Indeed it was argued in ref. [21] that this is a necessity in certain Higgs-boson jet-veto studies. Here it is not our intention to argue that all-order resummation of $\ln R^{2}$ enhanced terms is an absolute necessity: with a typical choice of $R=0.4,\left|\ln R^{2}\right| \simeq 2$, which is not a genuinely large number. However with increasing use of yet smaller $R$ values, it does become of interest to introduce techniques to carry out small- $R$ resummation. Furthermore, even for only moderately small $R$ values, a small- $R$ resummation can bring insight and understanding about the origins of higher-order corrections.

Logarithms of $R$ have been partially resummed before, in a soft approximation, for jet shapes [22]. Double logarithms, $\left(\alpha_{s} \ln R^{2} \ln p_{t, \mathrm{cut}}\right)^{n}$, and first subleading logarithms have been resummed for jet multiplicities [23] above some $p_{t}$ threshold, $p_{t, \text { cut }}$. Here, using an approach based on angular ordering, we show how to resum the leading logarithms of $R(\mathrm{LL})$, terms $\left(\alpha_{s} \ln R^{2}\right)^{n}$, for a wide range of jet observables, including the inclusive jet spectrum, the transverse momentum loss from a hard jet, jet veto probabilities, with results also for filtered and trimmed jets. ${ }^{1}$ In each case, we also include calculations of the coefficients of the first few orders of the perturbative expansion, which can give insight into the likely convergence of fixed-order perturbative calculations. ${ }^{2}$

\footnotetext{
${ }^{1}$ The approach that we use has a connection also with the problem of photon isolation in small cones, for which the structure of the leading log resummation and some phenomenological results were presented in ref. [24].

${ }^{2}$ Second-order small-R calculations have been performed in refs. [25, 26]. In the case of ref. [25], for
} 


\section{Jet definitions}

For concreteness we will work with jet algorithms from the generalised longitudinallyinvariant $k_{t}$ family, which includes the $k_{t}[2,27]$, Cambridge/Aachen [28, 29] and anti$k_{t}[30]$ algorithms. These algorithms introduce a distance measure between every pair of particles $i$ and $j$

$$
d_{i j}=\min \left(p_{t i}^{2 p}, p_{t j}^{2 p}\right) \frac{\Delta_{i j}^{2}}{R^{2}}
$$

and a distance measure between each particle and the beam,

$$
d_{i B}=p_{t i}^{2 p} .
$$

Here, $p_{t i}$ is the transverse momentum of particle $i, \Delta_{i j}^{2}=\left(y_{i}-y_{j}\right)^{2}+\left(\phi_{i}-\phi_{j}\right)^{2}$, where $y_{i}=\frac{1}{2} \ln \frac{E_{i}+p_{z i}}{E_{i}-p_{z i}}$ and $\phi_{i}$ are respectively the rapidity and azimuth of particle $i$. The parameter $p$ determines which jet algorithm one is using: $p=1,0,-1$ give respectively the $k_{t}$, Cambridge/Aachen and anti- $k_{t}$ algorithm. $R$ is the jet radius parameter.

The generalised $k_{t}$ algorithm proceeds by identifying the smallest of the $d_{i j}$ and $d_{i B}$. If it is a $d_{i j}$ then particles $i$ and $j$ are merged into a single new particle and all distances are updated. If it is a $d_{i B}$, then particle $i$ is called a "jet" and removed from the list of particles. It is straightforward to see that if a particle $i$ has no other particle $j$ within a distance $\Delta_{i j}<R$, then $d_{i B}$ will be smaller than any of the $d_{i j}$, and the only option for particle $i$ is for it to become a jet. On the other hand if there is a particle within $\Delta_{i j}<R$, then those two can merge. It is in this way that $R$ sets the angular scale on which particles can recombine into a single jet.

In order to carry out a leading-logarithmic resummation of $\left(\alpha_{s} \ln R^{2}\right)^{n}$ terms, we will be considering configurations of particles where the angles are strongly ordered, e.g. $\theta_{12} \gg$ $\theta_{23} \gg \theta_{34} \gg \ldots$, with $\theta_{i j}$ the angle between particles $i$ and $j$. For such configurations, all members of the generalised- $k_{t}$ family give identical jets, so we need only carry out a single resummation. The results will be valid also for infrared-safe cone algorithms such as SISCone [31] and also for $e^{+} e^{-}$variants of these algorithms, formulated directly in terms of energies and angles rather than $p_{t i}$ and $\Delta_{i j}$. In what follows, for simplicity, we will often simply use angle and energy variables.

\section{All-order leading-logarithmic resummation}

The basis of our resummation will be to start with a parton and consider the emissions from that parton at successively smaller angular scales. When we ask questions about (micro)jets with a radius $R$, it is equivalent at LL order to asking about the set of partons that is produced by the initial parton $i$ after allowing for all possible strongly ordered emissions down to angular scale $R$.

jet-vetos in Higgs production, we give comparisons in appendix B.1. Ref. [26] examined contributions to the resummation of thrust for small- $R$ jets, which do not appear to be directly comparable to the results derived here. 


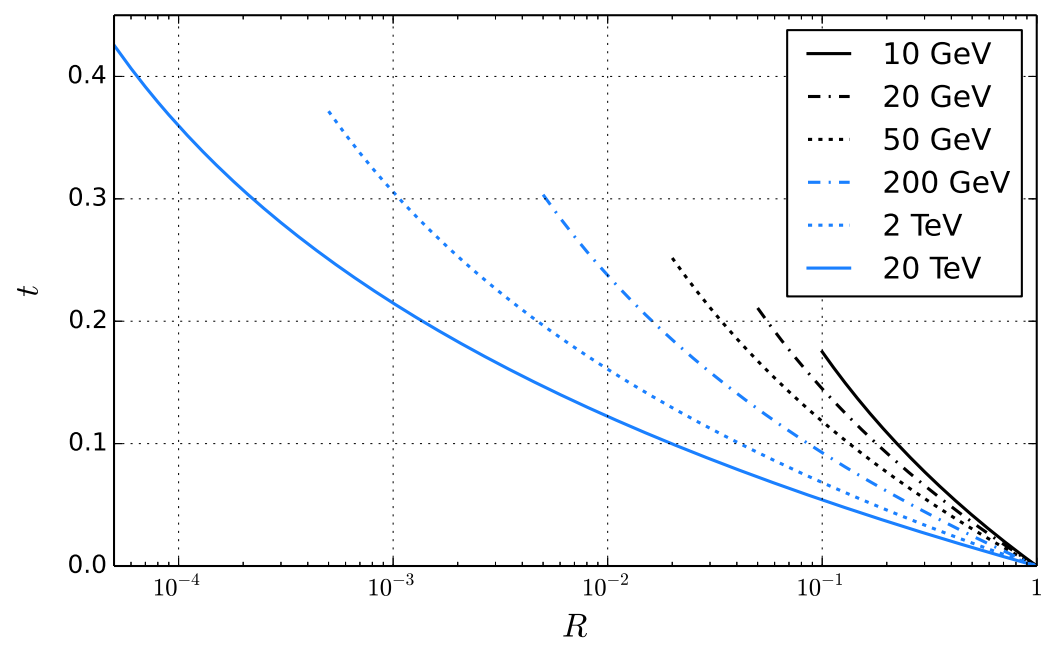

Figure 1. Evolution variable $t$ as a function of angular scale $R$ for $p_{t}=10,20,50,200,2000$ and $20000 \mathrm{GeV}$. Here $R$ is plotted down to $R p_{t}=1 \mathrm{GeV}$. For this, and for all other plots that involve a translation between $R$ and $t$, we use a one-loop coupling with 5 flavours such that $\alpha_{s}\left(M_{Z}\right)=0.1184$.

It will be convenient to introduce an evolution variable $t$ that corresponds to the integral over the collinear divergence, weighted with $\alpha_{s}$ at the appropriate renormalisation scale,

$$
t\left(R, p_{t}\right)=\int_{R^{2}}^{1} \frac{d \theta^{2}}{\theta^{2}} \frac{\alpha_{s}\left(p_{t} \theta\right)}{2 \pi}
$$

where $p_{t}$ here is the transverse momentum of the initial parton. ${ }^{3}$ The expansion of $t$ as a power series in $\alpha_{s}$ is

$$
t\left(R, p_{t}\right)=\frac{1}{b_{0}} \ln \frac{1}{1-\frac{\alpha_{s}\left(p_{t}\right)}{2 \pi} b_{0} \ln \frac{1}{R^{2}}}=\frac{1}{b_{0}} \sum_{n=1}^{\infty} \frac{1}{n}\left(\frac{\alpha_{s}\left(p_{t}\right) b_{0}}{2 \pi} \ln \frac{1}{R^{2}}\right)^{n}
$$

with $b_{0}=\frac{11 C_{A}-4 T_{R} n_{f}}{6}$. In a fixed coupling approximation, $t$ is simply $\frac{\alpha_{s}}{2 \pi} \ln \frac{1}{R^{2}}$.

The dependence of $t$ on the angular scale $R$ is shown in figure 1 for a range of $p_{t}$ values. The evolution variable is plotted over a range of $t$ such that $R p_{t} \geq 1 \mathrm{GeV}$, and we see that in most cases, typical values for $t$ are well below $t \sim 0.4$, which we will therefore take as an upper limit for the rest of this article. Two reference points that we will use throughout this article are $R=0.4$ and $R=0.2$, which correspond to $t \simeq 0.041$ and $t \simeq 0.077$ respectively for $p_{t}=50 \mathrm{GeV}$.

To understand the structure of emissions as one evolves in angle, we will make use of angular ordering [32,33]. This tells us that if an emission $i$ splits to $j$ and $k$ with opening angle $\theta_{j k}$, then any subsequent emission $\ell$ from $j$ on an angular scale $\theta_{j l} \ll \theta_{j k}$ is driven purely by the collinear divergence around $j$ and is independent of the properties of $k$ (and

\footnotetext{
${ }^{3}$ There is some freedom here on the choice upper limit in angle and on the exact scale of $\alpha_{s}$, but these do not matter at the LL accuracy that we are targeting.
} 
similarly with $j \leftrightarrow k) .{ }^{4}$ This will make it relatively straightforward to write an evolution equation that will encode the full set of potential emission configurations from an initial hard parton.

To do so, it will be helpful to introduce a generating functional (cf. textbooks such as $[32,33])$. Suppose that on some angular scale, defined by a $t$-value of $t_{1}$, we have a quark with momentum $x p_{t}$. We will define a generating functional $Q\left(x, t_{1}, t_{2}\right)$ that encodes the parton, or equivalently, microjet, content that one would observe if one now resolved that parton on an angular scale defined by $t_{2}>t_{1}$. The generating functional $Q\left(x, t_{1}, t_{2}\right)$ satisfies the condition

$$
Q(x, t, t)=q(x),
$$

where the $q(x)$ term indicates a $100 \%$ probability of finding just a quark with momentum $x p_{t}$. To first order in an expansion in $t_{2}-t_{1}$ (or equivalently order $\alpha_{s}$ ),

$$
Q\left(x, t_{1}, t_{2}\right)=q(x)+\left(t_{2}-t_{1}\right) \int_{0}^{1} d z p_{q q}(z)[q(z x) g((1-z) x)-q(x)]+\mathcal{O}\left(\left(t_{2}-t_{1}\right)^{2}\right)
$$

where the real $q \rightarrow q g$ splitting function is $p_{q q}(z)=C_{F}\left(\frac{1+z^{2}}{1-z}\right)$. Eq. (3.4) indicates that in addition to the state with just a quark, $q(x)$, at order $t$ there can also be states with both a quark and a gluon carrying respectively momentum $z x p_{t}$ and $(1-z) x p_{t}$; this is represented by $q(z x) g((1-z) x) .{ }^{5}$ As well as a generating functional $Q$ from an initial quark, we have one from an initial gluon, $G\left(x, t_{1}, t_{2}\right)$, with the property $G(x, t, t)=g(x)$.

For concreteness, the mean numbers of quark and gluon microjets of momentum $z p_{t}$, on an angular scale defined by $t$, produced from a quark of momentum $p_{t}$ are respectively

$$
\frac{d n_{q(z)}(t)}{d z}=\left.\frac{\delta Q(1,0, t)}{\delta q(z)}\right|_{\forall q(x)=1, g(x)=1}, \quad \frac{d n_{g(z)}(t)}{d z}=\left.\frac{\delta Q(1,0, t)}{\delta g(z)}\right|_{\forall q(x)=1, g(x)=1}
$$

where $\delta q(z)$ indicates a functional derivative. For $t=0$, eq. (3.5) consistently gives the expected result.

\subsection{Generating functional evolution equation}

Let us now formulate an evolution equation for the generating functionals. We first consider how to relate a quark generating function at initial scale 0 to one at an infinitesimal initial scale $\delta_{t}$ :

$$
\begin{aligned}
Q(x, 0, t)= & Q\left(x, \delta_{t}, t\right)\left(1-\delta_{t} \int d z p_{q q}(z)\right)+ \\
& +\delta_{t} \int d z p_{q q}(z)\left[Q\left(z x, \delta_{t}, t\right) G\left((1-z) x, \delta_{t}, t\right)\right] .
\end{aligned}
$$

\footnotetext{
${ }^{4}$ One caveat with angular ordering arises if one looks at azimuthal correlations. E.g., consider three particles such that $\theta_{23} \ll \theta_{12} \ll 1$, and define $\phi_{32}$ as the azimuthal angle of particle 3 around particle 2 ; then the distribution in $\phi_{32}$ is not necessarily uniform. In our study here, we will not be considering any observables that depend on the azimuthal angles, and so this issue does not need to be taken into account.

${ }^{5}$ It is important to remember, therefore, that $q(x)$ and $g(x)$ are therefore not parton distributions.
} 
The term on the first line involves the probability, in large round brackets, that the initial quark does not branch between scales 0 and $\delta_{t}$, so that the partonic content is given by that of a quark evolving from $\delta_{t}$ to $t$. The term on the second line involves the probability that there was a $q \rightarrow q g$ branching in the interval 0 to $\delta_{t}$, where the quark and gluon take fractions $z$ and $1-z$ of the original quark's momentum and the partonic content is now the combined content of the quark and the gluon, both evolving from $\delta_{t}$ to $t$ (this is represented by the product of generating functionals). At LL accuracy the generating functionals depend only on the difference of $t$ values, so we may replace $Q\left(x, \delta_{t}, t\right)=Q\left(x, 0, t-\delta_{t}\right)$. It is then straightforward to rewrite eq. (3.6) as a differential equation in $t$,

$$
\frac{d Q(x, t)}{d t}=\int d z p_{q q}(z)[Q(z x, t) G((1-z) x, t)-Q(x, t)],
$$

where we have introduced the shorthand notation $Q(x, t) \equiv Q(x, 0, t)$. One may proceed in a similar manner for gluons, giving

$$
\begin{aligned}
\frac{d G(x, t)}{d t}= & \int d z p_{g g}(z)[G(z x, t) G((1-z) x, t)-G(x, t)]+ \\
& +\int d z p_{q g}(z)[Q(z x, t) Q((1-z) x, t)-G(x, t)] .
\end{aligned}
$$

where the two further real splitting functions are

$$
\begin{aligned}
& p_{g g}(z)=2 C_{A}\left(\frac{z}{1-z}+\frac{1}{2} z(1-z)\right), \\
& p_{q g}(z)=n_{f} T_{R}\left(z^{2}+(1-z)^{2}\right) .
\end{aligned}
$$

Exploiting the $z \leftrightarrow(1-z)$ symmetry of eq. (3.8), we have written $p_{g g}(z)$ such that it has a divergence only for $z \rightarrow 1$. It will also be convenient to have defined the standard leadinglogarithmic splitting functions including the virtual terms, $P_{q q}(z)=p_{q q}(z)_{+}, P_{g q}(z)=$ $p_{q q}(1-z), P_{g g}(z)=p_{g g}(z)_{+}+p_{g g}(1-z)-\frac{2}{3} n_{f} T_{R} \delta(1-z)$ and $P_{q g}(z)=2 p_{q g}(z)$ (we sum over quarks and anti-quarks), with the usual definition of the plus prescription.

While eqs. (3.7) and (3.8) have been obtained by introducing an infinitesimal step of evolution at the beginning of the branching process, it is also possible to write an equation based on the addition of an infinitesimal step of evolution at the end of the branching. For a generating functional $F(t)$ that represents the evolution from any generic initial condition (i.e. not necessarily a single quark or a single gluon), the resulting equation reads

$$
\begin{aligned}
& \frac{d F(t)}{d t}=\int d x d z\left\{\frac{\delta F(t)}{\delta q(x)} p_{q q}(z)[q(z x) g((1-z) x)-q(x)]+\right. \\
& \left.+\frac{\delta F(t)}{\delta g(x)}\left[p_{g g}(z)(g(z x) g((1-z) x)-g(x))+p_{q g}(z)(q(z x) q((1-z) x)-g(x))\right]\right\} .
\end{aligned}
$$

The logic of this equation is that for each possible momentum fraction $x$, one considers all ways of extracting a quark or a gluon with that momentum fraction, $\delta_{q(x)} F$ or $\delta_{g(x)} F$, and then integrates over all allowed splittings. Eqs. (3.7) and (3.8) and eq. (3.10) are equivalent, and can be derived from a common starting point. Depending on the context, one or the other may be more convenient. 


\subsection{Fixed-order expansions for the generating functionals}

It is straightforward to solve the coupled pair of equations (3.7) and (3.8) order by order as a power expansion in $t$. Writing

$$
Q(x, t)=\sum_{n} \frac{t^{n}}{n !} Q_{n}(x), \quad G(x, t)=\sum_{n} \frac{t^{n}}{n !} G_{n}(x),
$$

and making use of the fact that it is sufficient to know just the result for $x=1$, we have

$$
\begin{aligned}
& Q_{0}(1)=q(1), \\
& \begin{aligned}
Q_{1}(1) & =\int d z p_{q q}(z)[q(z) g(1-z)-q(1)] \\
Q_{2}(1) & =\int d z d z^{\prime} p_{q q}(z)\left[p_{g g}\left(z^{\prime}\right)\left(q(z) g\left((1-z)\left(1-z^{\prime}\right)\right) g\left(z^{\prime}(1-z)\right)-g(1-z) q(z)\right)+\right. \\
& +p_{q q}\left(z^{\prime}\right)\left(g(1-z)\left(g\left(z\left(1-z^{\prime}\right)\right) q\left(z z^{\prime}\right)-q(z)\right)-g\left(1-z^{\prime}\right) q\left(z^{\prime}\right)+q(1)\right)+ \\
& \left.+p_{q g}\left(z^{\prime}\right)\left(q(z) q\left((1-z)\left(1-z^{\prime}\right)\right) q\left((1-z) z^{\prime}\right)-g(1-z) q(z)\right)\right],
\end{aligned}
\end{aligned}
$$

for the quark case, and

$$
\begin{aligned}
& G_{0}(1)=g(1), \\
& \begin{aligned}
& G_{1}(1)= \int d z\left[p_{g g}(z)(g(z) g(1-z)-g(1))+p_{q g}(z)(q(z) q(1-z)-g(1))\right], \\
& G_{2}(1)=\int d z d z^{\prime}\left[p _ { g g } ( z ) p _ { g g } ( z ^ { \prime } ) \left(g(1-z) g\left(z z^{\prime}\right) g\left(z\left(1-z^{\prime}\right)\right)-2 g(1-z) g(z)+\right.\right. \\
&\left.\quad+g(z) g\left((1-z)\left(1-z^{\prime}\right)\right) g\left(z^{\prime}(1-z)\right)-g\left(1-z^{\prime}\right) g\left(z^{\prime}\right)+g(1)\right)+ \\
& \quad+p_{g g}\left(z^{\prime}\right) p_{q g}(z)\left(g(1)-g\left(1-z^{\prime}\right) g\left(z^{\prime}\right)\right)+p_{q g}(z) p_{q g}\left(z^{\prime}\right)\left(g(1)-q\left(1-z^{\prime}\right) q\left(z^{\prime}\right)\right)+ \\
& \quad+p_{g g}(z) \\
& \quad p_{q g}\left(z^{\prime}\right)\left(g(1-z) q\left(z z^{\prime}\right) q\left(z\left(1-z^{\prime}\right)\right)-2 g(1-z) g(z)+\right. \\
&\left.\quad+g(z) q\left((1-z)\left(1-z^{\prime}\right)\right) q\left((1-z) z^{\prime}\right)-q\left(1-z^{\prime}\right) q\left(z^{\prime}\right)+g(1)\right)+ \\
& \quad+p_{q g}(z) \\
& \quad p_{q q}\left(z^{\prime}\right)\left(q(1-z) g\left(z-z z^{\prime}\right) q\left(z z^{\prime}\right)-2 q(1-z) q(z)\right. \\
&\left.\left.\quad+q(z) g\left((1-z)\left(1-z^{\prime}\right)\right) q\left((1-z) z^{\prime}\right)\right)\right]
\end{aligned}
\end{aligned}
$$

for the gluon case. These, and corresponding higher-order expansions, will be used to obtain the first few orders of the series in $t$ for a range of observables below. They can be used both analytically and numerically, by Monte Carlo integration over the $z, z^{\prime}$, with each term in the integrand corresponding to a specific partonic configuration.

\subsection{All-order reformulation}

Eqs. (3.7) and (3.8) can be equivalently stated as integral equations

$$
Q(x, t)=\Delta_{q}(t) Q(x, 0)+\int_{0}^{t} d t^{\prime} \Delta_{q}\left(t-t^{\prime}\right) \int_{\epsilon}^{1-\epsilon} d z p_{q q}(z) Q\left(z x, t^{\prime}\right) G\left((1-z) x, t^{\prime}\right)
$$


and

$$
\begin{aligned}
& G(x, t)=\Delta_{g}(t) G(x, 0)+\int_{0}^{t} d t^{\prime} \Delta_{g}\left(t-t^{\prime}\right) \int_{\epsilon}^{1-\epsilon} d z\left[p_{g g}(z) G(z x, t) G\left((1-z) x, t^{\prime}\right)+\right. \\
& \left.p_{q g}(z) Q(z x, t) Q\left((1-z) x, t^{\prime}\right)\right]
\end{aligned}
$$

where we have introduced Sudakov-like form factors:

$$
\begin{aligned}
& \Delta_{q}(t)=\exp \left(-t \int_{\epsilon}^{1-\epsilon} d z p_{q q}(z)\right) \\
& \Delta_{g}(t)=\exp \left(-t \int_{\epsilon}^{1-\epsilon} d z\left(p_{g g}(z)+p_{q g}(z)\right)\right) .
\end{aligned}
$$

The $\epsilon$ cutoffs serve to regularise the divergences in the splitting functions. In the limit $\epsilon \rightarrow 0$, the results for $Q(x, t)$ and $G(x, t)$ are independent of $\epsilon$.

The above expressions are suitable for Monte Carlo implementation as a recursive sequence of splittings, with the Sudakov-like $\Delta_{q / g}(t)$ factors acting as no-splitting probability distributions. We have used such a Monte Carlo implementation, which generates explicit partonic configurations, for the all-order results discussed below. ${ }^{6}$ One can similarly reformulate eq. (3.10), for which we again have a Monte Carlo implementation. It gives identical results.

For numerical purposes we usually take $\epsilon=10^{-3}$, which we find is sufficient in order to obtain percent-level accuracy.

Before continuing to the results, it is perhaps worth commenting on the relation between what we are calculating here and what is contained in parton-shower Monte Carlo programs. We have used an angle as our ordering variable; alternative variables used in some showers, such as relative transverse momentum or virtuality differ just by factors of $z$ and/or $1-z$ (and possibly an overall dimensionful constant). Because we only consider finite values of $z$ (neither arbitrarily small, nor arbitrarily close to 1), the impact of a factor of $z$ or $1-z$ in the choice of ordering variable is relevant only for terms beyond LL accuracy. Thus all parton showers should contain the small- $R$ leading logarithms that we are resumming here. One of the main differences between a parton shower and our calculation (apart from the much wider applicability of a shower) is that we can isolate a specific physical contribution, making it possible to obtain analytic results (e.g. for the expansion in powers of $\alpha_{s}$ ), physical insight and to straightforwardly combine results with other calculations.

\section{Results}

In this section we will show illustrative results for a few key observables of current relevance. The methods that we use can however be applied more generally.

\footnotetext{
${ }^{6}$ By "partonic configuration", we don't mean full 4-vector information, but instead a $z$ momentum fraction for each parton and a flavour label, quark or gluon.
} 


\subsection{Inclusive microjet observables}

The most basic collider jet observable is the inclusive jet spectrum, measured in the past years for example at HERA [34, 35], RHIC [5], the Tevatron [36, 37] and LHC [10, 38, 39]. It has been the subject of many phenomenological studies and calculations and is of considerable importance notably for constraining parton distribution functions. See for example recent progress in NNLO jet predictions [40, 41] and threshold resummation [42] and references therein.

Let us introduce the inclusive microjet fragmentation function: given a parton of flavour $i, f_{j / i}^{\text {incl }}(z, t)$ is the inclusive distribution of microjets of flavour $j$, at an angular scale defined by $t$, carrying a fraction $z$ of the parton's moment. Momentum conservation ensures that

$$
\sum_{j} \int d z z f_{j / i}^{\text {incl }}(z, t)=1
$$

In terms of the quantities introduced in section $3, f_{q / q}^{\text {incl }}(z, t)$ is for example nothing other than $d n_{q(z)}(t) / d z$ as obtained from the $Q(1, t)$ generating functional.

The inclusive microjet fragmentation function trivially satisfies a DGLAP-style equation

$$
\frac{d f_{j / i}^{\mathrm{incl}}(z, t)}{d t}=\sum_{k} \int_{z}^{1} \frac{d z^{\prime}}{z^{\prime}} P_{j k}\left(z^{\prime}\right) f_{k / i}^{\mathrm{incl}}\left(z / z^{\prime}, t\right),
$$

with an initial condition

$$
f_{j / i}^{\text {incl }}(z, 0)=\delta(1-z) \delta_{j i} .
$$

The inclusive microjet spectrum is particularly simple in that one does not need to make use of the full structure of the generating functional in order to obtain it. $^{7}$ Note that a similar result is a part also of the small- $R$ resummation of the fragmentation contribution to isolated photon production considered in ref. [24].

The solution to eq. (4.2) can be obtained using minor adaptations of standard DGLAP evolution codes, e.g. QCDNUM [43], QCD-Pegasus [44], HOPPET [45] or Apfel [46]. ${ }^{8}$ Alternatively one can use the Monte Carlo solution for the generating functional outlined in section 3, which is the choice we have made here.

The behaviour of the inclusive microjet fragmentation function when evolving to smaller angular scales is shown in figure 2 for $t=0.04,0.1,0.2$ and 0.3 . Here, and for all subsequent numerical results, we use $n_{f}=5$. The solid lines represent the inclusive microjet fragmentation function, in blue for an initial quark and in green for an initial gluon. The fragmentation functions are summed over the flavour of the microjets. One feature of the plots is a peak near $z=1$, showing the presence of the original parton with an almost unchanged momentum. As $t$ increases, that peak disappears, and does so more quickly for initial gluons than for initial quarks. Away from $z=1$, the fragmentation function for

\footnotetext{
${ }^{7}$ Though if one does wish to derive it from the generating functional, it is easiest to do so using eq. (3.10).

${ }^{8} \mathrm{An}$ evolution to an angular scale defined by $t$ is most straightforwardly mapped to a leading-logarithmic DIS evolution, using a $\delta(1-x)$ initial condition at some scale $Q_{0}$ and evolving to a higher scale $Q_{1}$ chosen such that $\int_{Q_{0}^{2}}^{Q_{1}^{2}} \frac{d Q^{2}}{Q^{2}} \frac{\alpha_{s}\left(Q^{2}\right)}{2 \pi}=t$. We have explicitly done this with HOPPET and verified that the results coincide with those from our Monte Carlo based solution.
} 

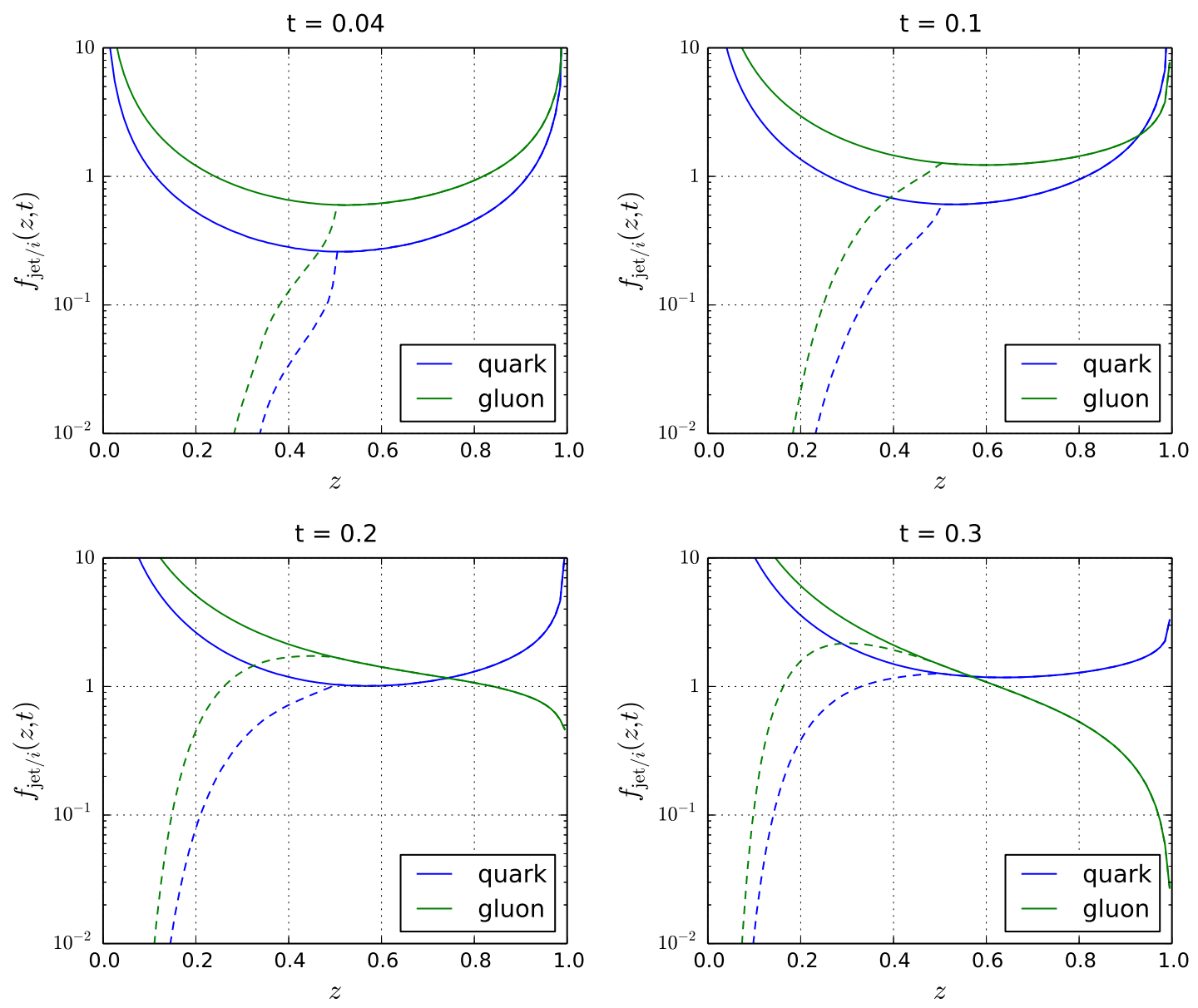

Figure 2. The solid lines show the inclusive microjet spectrum for initial quarks (blue) and initial gluons (green) using LL resummation of $\ln R^{2}$ enhanced terms. The dashed lines show the spectrum of the hardest microjet. They differ from the solid lines only for $z<\frac{1}{2}$. The four panes correspond to $t=0.04,0.1,0.2$ and 0.3 .

gluons is larger than that for quarks, as is to be expected given the larger colour factor. Finally at small $z$, there is a second peak, associated with production of multiple soft gluon microjets. The peak regions do not include resummation of logarithms of $1-z$ for $z$ near 1 , nor those of $z$ for small $z$. The resummation of double logarithms $\alpha_{s} \ln R^{2} \ln z$ (and the first tower of subleading terms) was discussed in ref. [23].

To examine the impact on a physical observable such as the inclusive jet spectrum in hadron collisions, it is necessary to convolute the inclusive microjet fragmentation function with the inclusive partonic spectrum from hard $2 \rightarrow 2$ scattering. Let us suppose the partonic spectrum for parton type $i$ is given by $d \sigma_{i} / d p_{t}$. Then the jet spectrum will be given by

$$
\frac{d \sigma_{\mathrm{jet}}}{d p_{t}}=\sum_{i} \int d p_{t}^{\prime} d z \frac{d \sigma_{i}}{d p_{t}^{\prime}} f_{\mathrm{jet} / i}^{\mathrm{incl}}(z, t) \delta\left(p_{t}-z p_{t}^{\prime}\right)=\left.\sum_{i} \int_{p_{t}} \frac{d z}{z} \frac{d \sigma_{i}}{d p_{t}^{\prime}}\right|_{p_{t}^{\prime}=p_{t} / z} f_{\mathrm{jet} / i}^{\mathrm{incl}}(z, t)
$$

where $f_{\text {jet } / i} \equiv \sum_{j} f_{j / i}$. If we assume that the partonic spectrum is dominated by a single 

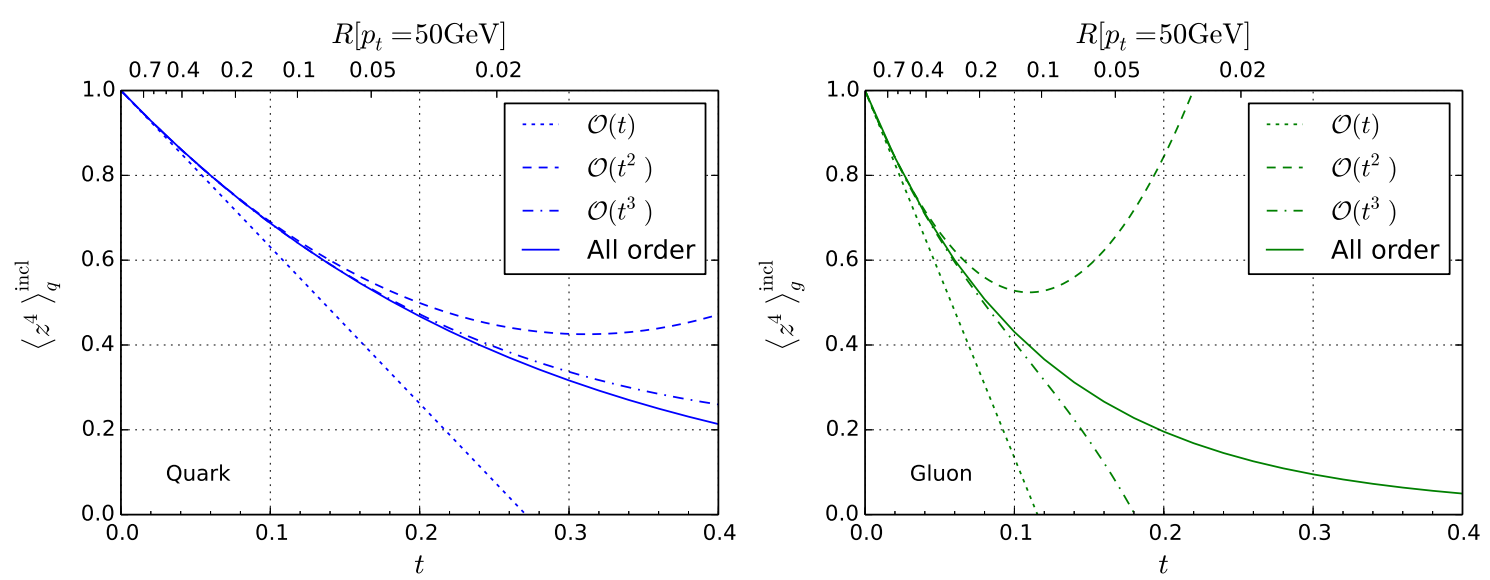

Figure 3. The result for $\left\langle z^{4}\right\rangle^{\text {incl }}$ at all orders as a function of $t$ (lower axis), together with the first 3 orders of its expansion in $t$, shown for initiating quarks (left) and gluons (right). The upper axis gives the correspond $R$ values for a jet with $p_{t}$ of order $50 \mathrm{GeV}$. The factor $\left\langle z^{4}\right\rangle^{\text {incl }}$, multiplied by a hard inclusive parton spectrum that goes as $p_{t}^{-5}$, gives the corresponding microjet spectrum.

flavour $i$ and its $p_{t}$ dependence locally is $d \sigma_{i} / d p_{t} \sim p_{t}^{-n}$, then one obtains the following multiplicative relation between the microjet and partonic spectra,

$$
\frac{d \sigma_{\mathrm{jet}}}{d p_{t}} \simeq \frac{d \sigma_{i}}{d p_{t}} \int_{0}^{1} d z z^{n-1} f_{\mathrm{jet} / i}^{\mathrm{incl}}(z, t)
$$

We will use the shorthand $\left\langle z^{n-1}\right\rangle_{i}^{\text {incl }}$ for the $z$ integral here. The all-order results are trivial to obtain analytically from the exponentiation of the matrix of moments of LO DGLAP splitting functions. The order-by-order expansion is likewise trivial. We accordingly refer the reader to eqs. (4.128) and (4.129) of ref. [33].

At the LHC, typical $n$ values range from about 4 at low $p_{t}$ to 7 or even higher at high $p_{t}$. The resummed $(n-1)^{\text {th }}$ moment of the inclusive microjet fragmentation function is shown for quarks and gluons in figure 3 , for $n=5$, together with the first few orders of the perturbative expansion in $t$. In this, and most of the other plots that follow, the lower $x$-axis shows the value of $t$, while the upper axis shows the corresponding value of $R$ for the case of a parton with $p_{t}=50 \mathrm{GeV}$.

The first observation that one makes from figure 3 is that small- $R$ effects can be substantial. For quark-induced jets with $R$ in the range 0.4 to 0.2 , they reduce the inclusivejet spectrum by $15-25 \%$. For gluon-induced jets the corresponding reductions are $30-50 \%$. These are substantial reductions in the cross-section, and help provide a motivation for wanting to understand small- $R$ effects.

One question one can ask is about the convergence of the perturbative series. For quark-induced jets the $\mathcal{O}(t)$ (i.e. NLO) result is accurate to within a couple of percent for $R=0.4$, while at $R=0.2$ one sees $5 \%$ differences relative to the all-order result. For gluoninduced jets, the $\mathcal{O}(t)$ result is off by about $10 \%$ for $R=0.4$, becomes inadequate around $R=0.3$ and pathological (negative) near $R=0.1$. Including the $\mathcal{O}\left(t^{2}\right)$ corrections (i.e. NNLO) brings agreement with all-orders to within a couple of percent for quark-initiated 
jets down to $R=0.1$; for gluon-initiated jets $\mathcal{O}\left(t^{2}\right)$ is adequate at $R=0.4$, but starts to deviate noticeably from the all-orders results below $R=0.3$.

Note that an expansion in $t$ is not directly equivalent to an expansion in $\alpha_{s}$, because the variable $t$ already resums the running-coupling contributions. As we will discuss at more length in a companion paper [47], an expansion in $\alpha_{s}$ appears more convergent. However, in light of the pattern of corrections as a power series in the natural evolution variable $t$, one wonders whether this apparently better convergence in $\alpha_{s}$ is to be trusted.

\subsection{Hardest microjet observables}

As well as the inclusive jet spectrum, it is common to ask questions about the hardest jet in an event, i.e. the jet with the largest transverse momentum. The hardest jet $p_{t}$ observable is relevant also whenever a jet veto is applied, since a veto is equivalent to a requirement that the hardest jet's $p_{t}$ be below some threshold. Given a parton with transverse momentum $p_{t}$, we define $f^{\text {hardest }}(z)$ to be the probability that the hardest resulting microjet carries a momentum $z p_{t} \cdot{ }^{9}$ Now instead of a momentum sum rule, we have a probability sum rule,

$$
\int_{0}^{1} d z f^{\text {hardest }}(z)=1
$$

In general, quantities involving the hardest microjet are more complicated than the inclusive quantities considered in section 4.1. The reason is that for an ensemble of microjets arising from multiple nested splittings, one has to consider all of the microjets together in order to determine which is the hardest. We have been able to carry out analytical calculations for configurations with up to three partons (order $t^{2}$ ), but have resorted to Monte Carlo methods to evaluate higher orders in $t$ and all-order results.

The all-order distribution of $f^{\text {hardest }}(z)$ is shown as dashed lines in figure 2 , for the same four $t$ values as the inclusive microjet fragmentation function. The inclusive and hardest microjet spectra are identical for $z>\frac{1}{2}$, since it is impossible to have more than one microjet with $z>\frac{1}{2}$. For small values of $t$, the hardest microjet spectrum shows a sharp transition at $z=\frac{1}{2}$, because below $z=\frac{1}{2}$ it can be non-zero only starting at order $t^{2}$. There is another transition at $z=\frac{1}{3}$, below which there are contributions only from order $t^{3}$ onwards. As $t$ increases, these transition points smoothen out significantly. The main difference between quark and gluon initiated jets is that the effects are more marked for the latter, as one would expect from the larger colour factor.

Next we consider various average properties of the hardest microjet. As well as numerical resummed results, we will also provide the coefficients of the first few orders of the power series in $t$, which we write as

$$
\mathcal{O}(t)=\sum_{n} \frac{t^{n}}{n !} c_{n}[\mathcal{O}]
$$

for a general observable $\mathcal{O}$. While we have analytical results for $c_{2}$, for the sake of conciseness we will just quote numerical values in this section, separately for each colour factor. The corresponding full analytical expressions are given in appendix A.

\footnotetext{
${ }^{9}$ We should really write $f^{\text {hardest }}(z, t)$, but in most cases drop the $t$ argument for compactness.
} 


\subsubsection{Hardest microjet $\langle\Delta z\rangle$}

A typical context in which the average fractional energy loss from a jet, $\langle\Delta z\rangle$, is relevant is in the study of the difference in $p_{t}$ between a $Z$-boson and the leading jet in $Z+$ jet events. This kind of quantity is used for jet calibration [3,4]. It is also relevant for example in jet-photon balance studies in heavy-ion collisions [48, 49].

The average fractional transverse-momentum difference between the hardest microjet and the initial parton is given by

$$
\langle\Delta z\rangle^{\text {hardest }} \equiv \int_{0}^{1} d z f^{\text {hardest }}(z)(z-1) .
$$

For an initial quark we find

$$
\begin{aligned}
&\langle\Delta z\rangle_{q}^{\text {hardest }}= C_{F} t\left(\frac{3}{8}-2 \ln 2\right)+ \\
&+\frac{t^{2}}{2}\left(-0.467188 C_{A} C_{F}+1.62588 C_{F}^{2}-0.0710364 C_{F} n_{f} T_{R}\right)+ \\
&+\frac{t^{3}}{6}\left(-2.33574(2) C_{F}^{3}+0.67962(2) C_{A}^{2} C_{F}+0.11881(2) C_{A} C_{F}^{2}+0.416131(6) C_{A} C_{F} n_{f} T_{R}-\right. \\
&\left.\quad-0.204121(5) C_{F}^{2} n_{f} T_{R}+0.0473591(7) C_{F} n_{f}^{2} T_{R}^{2}\right)+\mathcal{O}\left(t^{4}\right), \quad \text { (4.9) }
\end{aligned}
$$

while the case of an initiating gluon yields

$$
\begin{gathered}
\langle\Delta z\rangle_{g}^{\text {hardest }}=t\left[-\frac{7}{48} n_{f} T_{R}+C_{A}\left(\frac{43}{96}-2 \ln 2\right)\right]+ \\
+\frac{t^{2}}{2}\left(0.962984 C_{A}^{2}+0.778515 C_{A} n_{f} T_{R}-0.50674 C_{F} n_{f} T_{R}+0.0972222 n_{f}^{2} T_{R}^{2}\right)+ \\
+\frac{t^{3}}{6}\left(-1.11718(2) C_{A}^{3}-1.557542(7) C_{A}^{2} n_{f} T_{R}+0.375492(7) C_{A} C_{F} n_{f} T_{R}+0.75869(1) C_{F}^{2} n_{f} T_{R}-\right. \\
\left.\quad-0.635406(3) C_{A} n_{f}^{2} T_{R}^{2}+0.305404(3) C_{F} n_{f}^{2} T_{R}^{2}-0.0648152(4) n_{f}^{3} T_{R}^{3}\right)+\mathcal{O}\left(t^{4}\right) .
\end{gathered}
$$

In figure 4 we can see the all-order results for the hardest microjet $\Delta z$, along with the fixed order expansion expressed in equations (4.9) and (4.10) truncated at the first, second and third powers in $t$. For quark-induced jets, the fractional energy loss is in the range $5-10 \%$ for $R=0.4-0.2$, while for gluon-induced jets it is in the range $10-20 \%$.

One feature of figure 4 is that one immediately notices a significantly better convergence than in figure 3 . This is because the $z^{4}$ weighting in figure 3 amplifies the impact of higher orders. On the other hand jet momenta tend to be measured with much higher accuracy $(\sim 1 \%[3,4])$ than steeply falling jet spectra, so one targets higher relative accuracy for $\langle\Delta z\rangle^{\text {hardest }}$. Quite high (percent-level) accuracy for the phenomenologically relevant range of $t$ is obtained even at order $t$ in the case of quark jets. However, for gluon jets $\mathcal{O}(t)$ is probably inadequate at $R=0.3$ and below. Going to order $t^{2}$ is probably sufficient for gluon jets down to $R=0.15$. As noted in the case of the inclusive jet spectrum an expansion in $t$ does not directly correspond to an expansion in $\alpha_{s}$, so further cross-checks on the validity of fixed-order calculations would be needed on a case-by-case basis. 

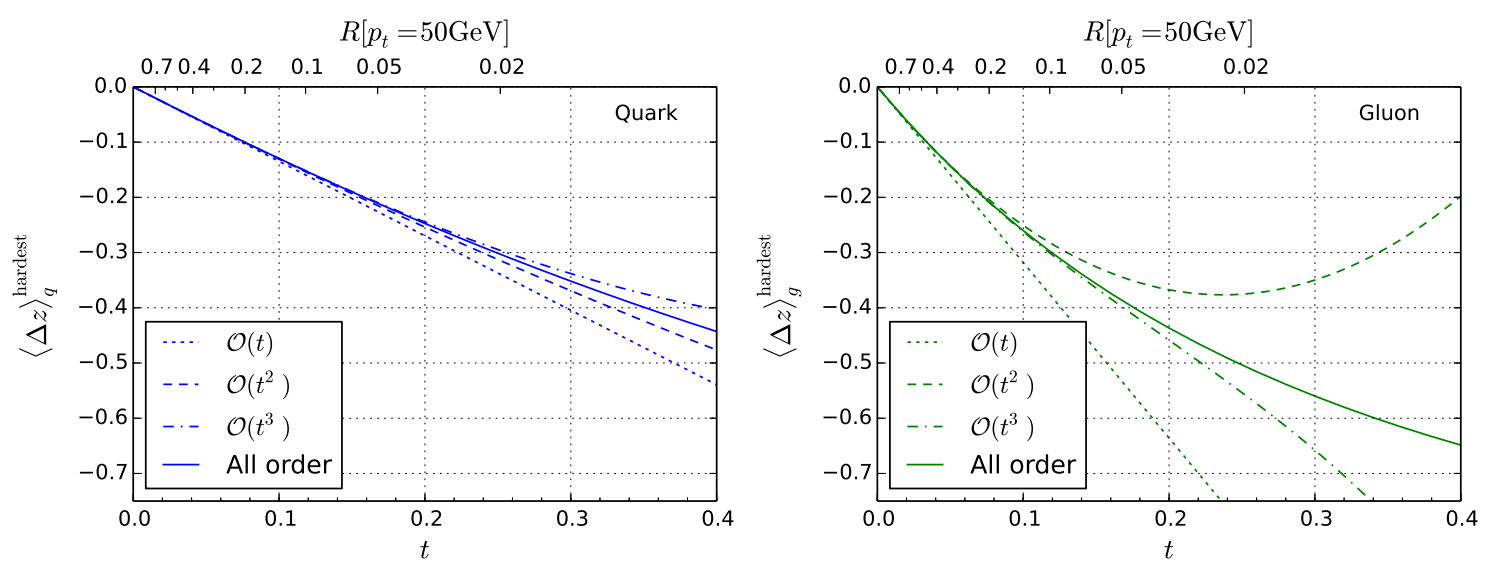

Figure 4. Average hardest microjet $\Delta z$, shown as a function of $t$ for quark-induced (left) and gluon-induced (right) jets. Resummed results are represented as solid lines. The first three orders in $t$ are represented as dotted, dashed and dash-dotted lines respectively.

\subsubsection{Jet-veto resummations and $\langle\ln z\rangle$}

Jet veto resummations are one of the contexts in which the potential need for all-order small- $R$ corrections has been raised [21]. In this context, as we shall derive below, the relevant quantity is $\langle\ln z\rangle^{\text {hardest }}$.

Let us first recall the core structure of a jet-veto resummation for finite- $R$ jets. As we shall see, when considering logarithms of the jet veto scale, it will be sufficient for our purposes to work at leading (double) logarithmic accuracy. This helps eliminate numerous complications such as those related to parton distribution functions. Assuming a process with a hard scale $Q$ and two incoming partons with colour factor $C\left(C_{F}\right.$ for quarks, $C_{A}$ for gluons), the probability of there being no gluons emitted above a scale $p_{t}$ is given by

$$
P(\text { no primary-parton veto })=\sum_{n=0}^{\infty} \frac{1}{n !} \prod_{i=1}^{n}\left[\int^{Q} \frac{d k_{t i}}{k_{t i}} \bar{\alpha}_{s}\left(k_{t i}\right) 2 \ln \frac{Q}{k_{t i}}\left(-1+\Theta\left(p_{t}-k_{t i}\right)\right)\right],
$$

where $\bar{\alpha}_{s}\left(k_{t}\right) \equiv 2 \alpha_{s}\left(k_{t}\right) C / \pi$. In the factor $\left(-1+\Theta\left(k_{t i}-p_{t}\right)\right)$, the $\Theta$-function corresponds to the veto on partons above a scale $p_{t}$, while the term -1 accounts for virtual corrections. The factor $2 \ln \frac{Q}{k_{t}}$ corresponds to the kinematically allowed range of rapidities for a gluon with transverse momentum $k_{t}$ (in a leading logarithmic approximation for the $p_{t}$ veto resummation). It is straightforward to see that eq. (4.11) corresponds to an exponential,

$$
P(\text { no primary-parton veto })=\exp \left[-\int_{p_{t}}^{Q} \frac{d k_{t}}{k_{t}} \bar{\alpha}_{s}\left(k_{t}\right) 2 \ln \frac{Q}{k_{t}}\right] .
$$

Defining $L \equiv \ln Q / p_{t}, \ln P$ contains "leading (double) logarithmic" terms $\alpha_{s}^{n} L^{n+1}$. The jet veto efficiency in Higgs and Drell-Yan production is currently known to NNLL accuracy in this language, i.e. $\alpha_{s}^{n} L^{n-1}$ [50-54]. The papers by the Becher et al. and the Stewart et al. groups include subsets of terms beyond NNLL, while heavy-quark effects in the $g g H$ interaction have been discussed in ref. [55]. 
To include small- $R$ corrections, one needs to modify eq. (4.11) to account for the fact that each of the partons $i=1 \ldots n$ will fragment into multiple microjets, and for each of those partons the veto now applies to the resulting hardest microjet. Thus for each emission in eq. (4.11), we integrate over the probability distribution for the momentum fraction of the hardest resulting microjet,

$$
\begin{aligned}
P(\text { no microjet veto })=\sum_{n=0}^{\infty} & \frac{1}{n !} \prod_{i=1}^{n}\left[\int^{Q} \frac{d k_{t i}}{k_{t i}} \bar{\alpha}_{s}\left(k_{t i}\right) 2 \ln \frac{Q}{k_{t i}} \times\right. \\
& \left.\times \int_{0}^{1} d z_{i} f^{\text {hardest }}\left(z_{i}, t\left(R, k_{t i}\right)\right)\left(-1+\Theta\left(p_{t}-z_{i} k_{t i}\right)\right)\right],
\end{aligned}
$$

where we have made the $t\left(R, k_{t i}\right)$ argument in $f^{\text {hardest }}\left(z_{i}, t\left(R, k_{t i}\right)\right)$ explicit, because of the importance of making the right scale choice for the definition of $t$ (cf. also eq. (3.1)). Given eq. (4.6), it is immaterial to the result whether the integration over $z$ takes place outside the large round brackets or inside, applied just to the $\Theta$-function. As before, we now write the result as an exponential,

$$
\begin{aligned}
& P(\text { no microjet veto })=\exp \left[-\int^{Q} \frac{d k_{t}}{k_{t}} \bar{\alpha}_{s}\left(k_{t}\right) 2 \ln \frac{Q}{k_{t}} \times\right. \\
& \left.\times \int_{0}^{1} d z f^{\text {hardest }}\left(z, t\left(R, k_{t}\right)\right)\left(\Theta\left(k_{t}-p_{t}\right)+\Theta\left(z k_{t}-p_{t}\right)-\Theta\left(k_{t}-p_{t}\right)\right)\right] .
\end{aligned}
$$

To help us perform the calculation, we have added and subtracted a term $\Theta\left(k_{t}-p_{t}\right)$. Then one considers the first $\Theta$ function separately from the second and third. For the first one, the $z$ integration can be performed trivially and one obtains the primary-parton result, eq. (4.12). For the remaining pair of $\Theta$-functions, we first evaluate the $k_{t}$ integral: since $k_{t}$ is being evaluated over a limited range, we can replace $\bar{\alpha}_{s}\left(k_{t}\right)$ with $\bar{\alpha}_{s}\left(p_{t}\right)$, and similarly in the $\ln Q / k_{t}$ and $t\left(R, k_{t}\right)$ factors. The terms that we neglect as a result of this are suppressed by one logarithm of $\ln Q / p_{t}$. We therefore have the following small- $R$ correction to the jet veto efficiency

$$
\mathcal{U} \equiv \frac{P(\text { no microjet veto })}{P(\text { no primary-parton veto })}=\exp \left[-2 \bar{\alpha}_{s}\left(p_{t}\right) \ln \frac{Q}{p_{t}} \int_{0}^{1} d z f^{\text {hardest }}\left(z, t\left(R, p_{t}\right)\right) \ln z\right] .
$$

This $R$-dependent correction generates a series of terms $\alpha_{s}^{m+n}(Q) L^{m} \ln ^{n} R^{2}$, while we have neglected terms suppressed by one or more powers of either $L=\ln Q / p_{t}$ or $\ln R^{2}$.

Eq. (4.15) shows that the key quantity for the small- $R$ part of the resummation is the first logarithmic moment of $f^{\text {hardest }}(z)$

$$
\langle\ln z\rangle^{\text {hardest }} \equiv \int_{0}^{1} d z f^{\text {hardest }}(z) \ln z .
$$

It is the logarithmic moment of the microjet spectrum from initial gluons that is relevant here, since the $i=1 \ldots n$ partons in eq. (4.13) are all gluons (regardless of whether the jet 

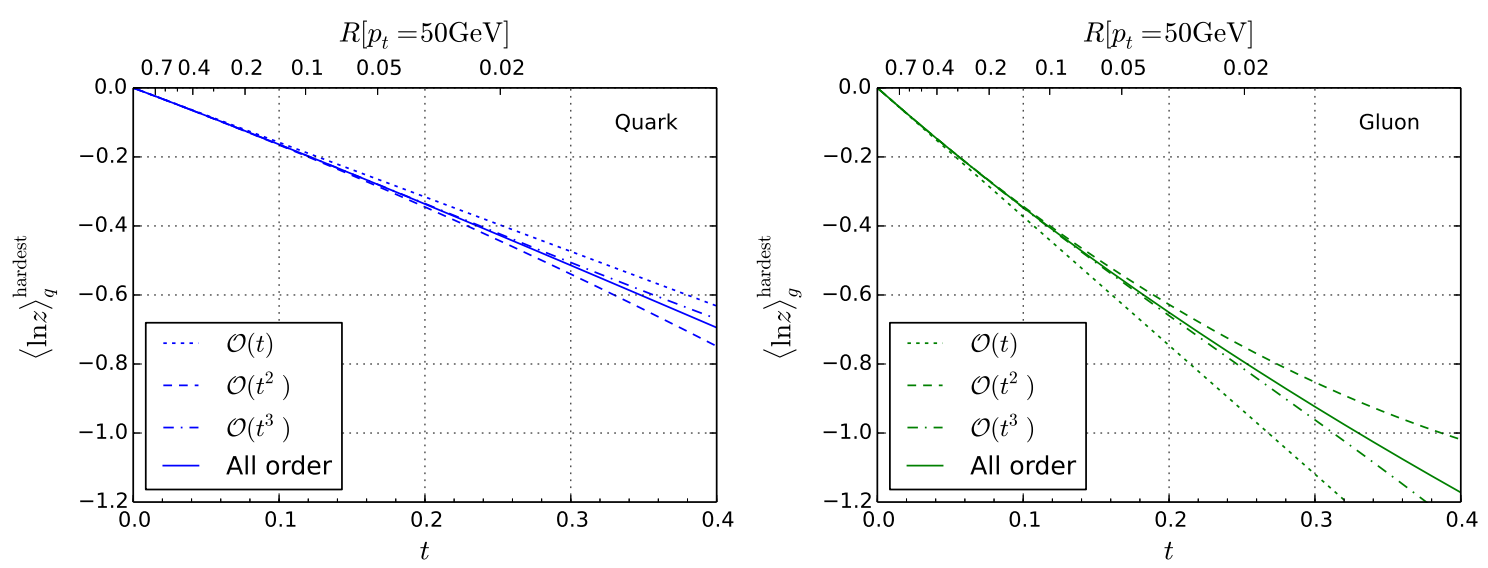

Figure 5. Average of the hardest microjet $\ln z$, as a function of $\mathrm{t}$, shown separately for quarkinduced (left) and gluon-induced (right) jets. The resummed results are represented as solid lines. The first three orders in $t$ are represented as dotted, dashed and dash-dotted lines respectively.

\begin{tabular}{|cccccc|}
\hline $\mathcal{O}$ & $c_{1}$ & $c_{2}$ & $c_{3}$ & $c_{4}^{\text {fit }}$ & $c_{5}^{\text {fit }}$ \\
\hline$\langle\ln z\rangle_{q}^{\text {hardest }}$ & -1.58 & -1.46 & 7.43 & -34.07 & 105.62 \\
$\langle\ln z\rangle_{g}^{\text {hardest }}$ & -3.73 & 5.92 & -24.20 & 133.55 & -478.55 \\
\hline
\end{tabular}

Table 1. Results of a fit to the all-order determination of $\langle\ln z\rangle^{\text {hardest }}$; the fit function is of the form $c_{1} t+c_{2} \frac{t^{2}}{2 !}+c_{3} \frac{t^{3}}{3 !}+c_{4}^{\text {fit }} \frac{t^{4}}{4 !}+c_{5}^{\text {fit }} \frac{t^{5}}{5 !}$ where the $c_{1}, c_{2}$ and $c_{3}$ coefficients are fixed to the values given in eqs. (A.5) and (4.17), while $c_{4}^{\text {fit }}$ and $c_{5}^{\text {fit }}$ have been fitted. The fit was performed in the range $0<t<0.4$ and has an accuracy of significantly better than $1 \%$ for $\langle\ln z\rangle^{\text {hardest }}$. The fit values for the fourth and fifth order coefficients are not to be taken as robust determinations of those coefficients, but simply as values whose use in the truncated sum gives good agreement with the all-order result.

veto is applied to a $q \bar{q}$ or gluon-fusion process). The first three orders of its expansion in $t$ are

$$
\begin{aligned}
& \langle\ln z\rangle_{g}^{\text {hardest }}=t\left[\frac{1}{72} C_{A}\left(131-12 \pi^{2}-132 \ln 2\right)+\frac{1}{36} n_{f} T_{R}(-23+24 \ln 2)\right]+ \\
& \quad+\frac{t^{2}}{2}\left(0.206672 C_{A}^{2}+0.771751 C_{A} n_{f} T_{R}-0.739641 C_{F} n_{f} T_{R}+0.117861 n_{f}^{2} T_{R}^{2}\right)+ \\
& +\frac{t^{3}}{6}\left(-0.20228(4) C_{A}^{3}-0.53612(2) C_{A}^{2} n_{f} T_{R}-0.062679(8) C_{A} C_{F} n_{f} T_{R}+0.54199(2) C_{F}^{2} n_{f} T_{R}-\right. \\
& \left.\quad-0.577215(3) C_{A} n_{f}^{2} T_{R}^{2}+0.431055(4) C_{F} n_{f}^{2} T_{R}^{2}-0.0785743(5) n_{f}^{3} T_{R}^{3}\right)+\mathcal{O}\left(t^{4}\right) .
\end{aligned}
$$

The order $t$ term of eq. (4.17), when incorporated into eq. (4.15), gives an $\alpha_{s}^{2} L \ln R^{2}$ term whose coefficient agrees with that given in refs. [51, 53, 54]. Full analytical results for the $\mathcal{O}\left(t^{2}\right)$ term are to be found in appendix A.2, as are analytical and numerical results for the corresponding logarithmic moment for quark fragmentation (potentially of interest for vetoes on initial state $g \rightarrow q \bar{q}$ splittings).

The all-order result for $\langle\ln z\rangle_{g}^{\text {hardest }}$ (and quark counterpart) is shown as a function of $t$ in figure 5, together with its expansion in $t$. The small- $R$ effects in the gluon-induced case are in the range $15 \%$ to a little over $25 \%$ for $R=0.2-0.4$, i.e. slightly larger than 
for $\langle\Delta z\rangle_{g}^{\text {hardest }}$. One notes the remarkably good convergence: for example at $t=0.1$, for $\langle\Delta z\rangle_{g}^{\text {hardest }}$ the difference between the $\mathcal{O}(t)$ and all-order results was roughly $25 \%$; in contrast for $\langle\ln z\rangle_{g}^{\text {hardest }}$ the difference is $7 \%$. It is not clear to us if there is a fundamental reason why this should be the case.

For practical use it is useful to have a parametrisation of the all-order result. Given the good convergence of the series, this can be obtained in the range $t<0.4$ simply by fitting additional $c_{4}$ and $c_{5}$ coefficients to the all-order curve. The results of the fit are given in table 1 and they allow one to reproduce the all-order result to an accuracy of better than $1 \%$. The actual values of the coefficients themselves are, however, not guaranteed to be accurate since they may in part be absorbing contributions from yet higher order terms. A study of the phenomenological impact of the small- $R$ resummation will be given elsewhere [47].

We note that a numerical calculation for the $\alpha_{s}^{3} L \ln ^{2} R^{2}$ term in the case of the jet veto for $g g \rightarrow H$ production was given in ref. [25]. At first sight it appears to disagree with our analytical result and after consultation with the authors they identified an issue in their treatment of $R$-dependent running coupling-related terms. The detailed comparison and discussion is to be found in appendix B.1. In appendix B.2 we present a cross-check of our calculational approach specifically for the second-order contribution to $f_{\text {hardest }}(z)$ for quark-induced jets, as obtained through a comparison to the Event 2 NLO program [56].

\subsubsection{Jet flavour}

The flavour of jets is a subject that is conceptually interesting and obtaining a better handle on jet flavour is potentially also of considerable practical use. ${ }^{10}$ As discussed at length in ref. [57], the definition of jet flavour is a subtle question. However, in the leadinglogarithmic collinear limit in which we work here, those subtleties disappear, essentially because they are related to soft radiation.

The question that we ask in this subsection is the following: given a quark (gluon) parton, how likely is it that the resulting hardest microjet will have the flavour of a gluon (quark). This is relevant, for example, when considering the performance of quark/gluon tagging algorithms, whether theoretically [58-61] or experimentally [62,63], since one is often assuming that the flavour of the selected (hardest) jet is identical to that of the underlying hard scattering.

To answer this question, we extend $f^{\text {hardest }}(z)$ to have flavour indices: $f_{a / b}^{\text {hardest }}(z)$ is the differential distribution in $z$ of hardest microjets of flavour $a$ given an initiating parton of flavour $b$. The overall probability, $\mathcal{P}(a \mid b)$ of producing a hardest microjet of flavour $a$, given an initial parton of flavour $b$, is

$$
\mathcal{P}(a \mid b)=\int_{0}^{1} d z f_{a / b}^{\text {hardest }}(z) .
$$

\footnotetext{
${ }^{10}$ Furthermore, jet flavour has seen extensive discussion in the literature, so much so that, for example, ref. [57] was able to identify over 350 articles with the terms "quark jet" or "gluon jet" in their title.
} 

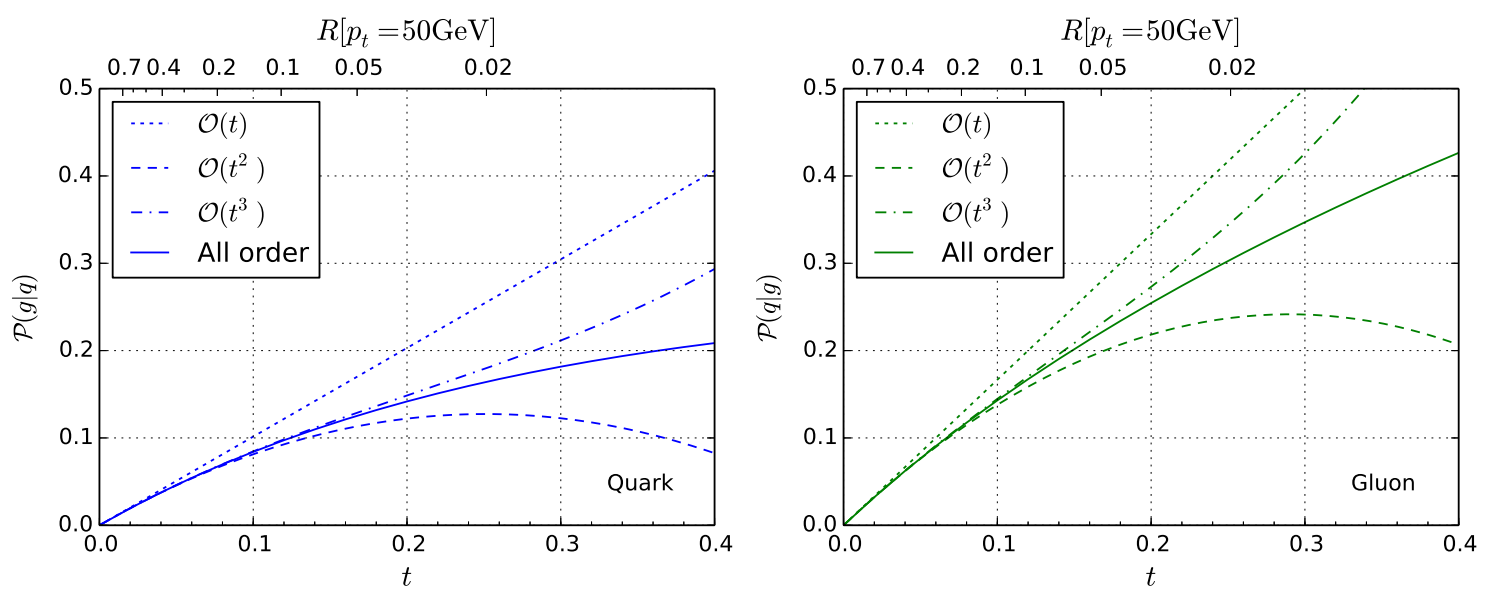

Figure 6. Hardest microjet flavour change probability as a function of $t$. The left-hand plot shows the probability for a quark-parton to emerge as a gluon microjet, while the right-hand plot shows the opposite flavour-changing probability. Resummed results are shown as solid lines, while the first three orders in $t$ are represented as dotted, dashed and dash-dotted lines respectively.

The two main cases of interest are

$$
\begin{aligned}
\mathcal{P}(g \mid q)=C_{F} t\left(\ln 4-\frac{5}{8}\right)+ & \frac{t^{2}}{2}\left(-0.610848 C_{A} C_{F}+0.0519619 C_{F}^{2}-0.50753 C_{F} n_{f} T_{R}\right)+ \\
+\frac{t^{3}}{6}\left(-1.112(6) C_{F}^{3}+\right. & 0.505(8) C_{A}^{2} C_{F}+0.92(2) C_{A} C_{F}^{2}+0.89157(6) C_{A} C_{F} n_{f} T_{R}- \\
& \left.-0.0903(1) C_{F}^{2} n_{f} T_{R}+0.338360(6) C_{F} n_{f}^{2} T_{R}^{2}\right)+\mathcal{O}\left(t^{4}\right),
\end{aligned}
$$

and

$$
\begin{aligned}
\mathcal{P}(q \mid g)= & \frac{2}{3} n_{f} T_{R} t+\frac{t^{2}}{2}\left(-0.201036 C_{A} n_{f} T_{R}-0.438979 C_{F} n_{f} T_{R}-0.444444 n_{f}^{2} T_{R}^{2}\right)+ \\
& +\frac{t^{3}}{6}\left(1.0498(2) C_{A}^{2} n_{f} T_{R}-0.10560(6) C_{A} C_{F} n_{f} T_{R}-0.2537(1) C_{F}^{2} n_{f} T_{R}+\right. \\
+ & \left.0.536081(8) C_{A} n_{f}^{2} T_{R}^{2}+0.585304(6) C_{F} n_{f}^{2} T_{R}^{2}+0.2962952(5) n_{f}^{3} T_{R}^{3}\right)+\mathcal{O}\left(t^{4}\right) .
\end{aligned}
$$

Analytical expressions for the $t^{2}$ coefficients are given in appendix A.3. The all-order results are shown in figure 6 . One sees for example that for $R=0.4$, a quark (gluon) has a $4 \%$ (6\%) probability of becoming a differently flavoured microjet; for $R=0.2$ the corresponding numbers are $7 \%(12 \%)$. These numbers are subject to substantial higher-order (and jetand flavour-definition related) uncertainties, but they give an order of magnitude for the maximal jet-flavour purity that can be obtained in samples generated from flavour-pure partonic samples.

There are other questions that may also be interesting to ask about jet flavour. For example one might investigate it also in the context of inclusive jet measurements, to identify how the flavour composition in a steeply-falling hard-scattering spectrum is modified in the resulting microjet spectrum. It would also be conceptually interesting (though perhaps not very physically relevant) to investigate the limit of asymptotically large $t$, where the ratio 
of quark to gluon microjets might be expected to tend to a fixed constant, independently of the whether the initial parton is a quark or gluon.

\subsection{Multi (sub)jet observables}

A number of methods developed for boosted electroweak and top-quark tagging naturally involve small-radius subjets, as discussed in various reviews [17-20]. These are used in part because the boost collimates the heavy-object decay products and associated radiation and in part to mitigate the impact of the very substantial pileup that is present at the LHC.

Here we will consider general purpose "grooming" approaches, filtering [14] and trimming [15], which, for their general ability to remove pileup, could have applications also beyond boosted-objected studies. In particular, it is interesting to ask how well filtered and trimmed jets maintain the momentum of an original parton. Similar considerations may apply also to the idea of building large jets from small jets [16].

\subsubsection{Filtering}

In filtering, one takes a jet clustered with an initial radius $R_{0}$, reclusters its constituents on a smaller angular scale, $R_{\text {filt }}<R_{0}$, and then discards all but the $n$ hardest subjets. Whereas $t$ in the previous sections was defined as being $\frac{\alpha_{s}}{2 \pi} \ln \frac{1}{R^{2}}$, plus higher orders from the running coupling, we now imagine taking a large-radius original jet, $R_{0}=\mathcal{O}(1)$ and processing it with a small filtering radius, with $t$ defined in terms of the filtering radius, $t \simeq \frac{\alpha_{s}}{2 \pi} \ln \frac{1}{R_{\text {filt }}^{2}}$, again plus higher orders from the running coupling. More generally, i.e. also for small $R_{0}, t \simeq \frac{\alpha_{s}}{2 \pi} \ln \frac{R_{0}^{2}}{R_{\text {filt }}^{2}}$ plus higher orders, and the quantities we work out here will then relate the filtered jet to the original jet rather than to the original parton.

We define $f^{k \text {-hardest }}(z)$ to be the probability that the $k$-th hardest subjet carries a momentum fraction $z$ of the initial parton (or large- $R$ jet). We can then express the energy loss between the filtered jet and the initial parton as

$$
\langle\Delta z\rangle^{\text {filt }, n}=\left[\sum_{k=1}^{n} \int d z z f^{k \text {-hardest }}(z)\right]-1 .
$$

The total energy loss when taking the sum of the $n=2$ hardest microjets is, for the case of an initiating quark

$$
\begin{aligned}
& \langle\Delta z\rangle_{q}^{\mathrm{filt}, 2}=\frac{t^{2}}{2}\left(-1.152 C_{A} C_{F}-3.15229 C_{F}^{2}-0.175607 C_{F} n_{f} T_{R}\right)+ \\
& +\frac{t^{3}}{6}\left(24.23(3) C_{F}^{3}+0.82448(2) C_{A}^{2} C_{F}+6.2567(2) C_{A} C_{F}^{2}+0.893365(6) C_{A} C_{F} n_{f} T_{R}+\right. \\
& \left.+0.30444(2) C_{F}^{2} n_{f} T_{R}+0.1170718(9) C_{F} n_{f}^{2} T_{R}^{2}\right)+\mathcal{O}\left(t^{4}\right),
\end{aligned}
$$

while for an initial gluon, we find

$$
\begin{aligned}
&\langle\Delta z\rangle_{g}^{\mathrm{filt}, 2}= \frac{t^{2}}{2}\left(-3.88794 C_{A}^{2}-0.5029 C_{A} n_{f} T_{R}-0.505401 C_{F} T_{R} n_{f}\right)+ \\
&+\frac{t^{3}}{6}\left(27.258(8) C_{A}^{3}+8.7362(2) C_{A}^{2} n_{f} T_{R}-0.59419(2) C_{A} C_{F} n_{f} T_{R}+\right. \\
&\left.+0.72083(2) C_{F}^{2} n_{f} T_{R}+0.740071(4) C_{A} n_{f}^{2} T_{R}^{2}+0.264690(3) C_{F} n_{f}^{2} T_{R}^{2}\right)+\mathcal{O}\left(t^{4}\right) .
\end{aligned}
$$


The full analytical results for the coefficients of $t^{2}$ are given in appendix A.4. If we consider the $n=3$ hardest subjets, then the first non-vanishing term is the $t^{3}$ coefficient, and we find, for an initial quark

$$
\begin{aligned}
\langle\Delta z\rangle_{q}^{\mathrm{filt}, 3} & =\frac{t^{3}}{6}\left(-17.02(3) C_{F}^{3}-5.0299(2) C_{A}^{2} C_{F}-18.9494(9) C_{A} C_{F}^{2}\right. \\
& \left.-0.643983(4) C_{A} C_{F} n_{f} T_{R}-3.1521(1) C_{F}^{2} n_{f} T_{R}\right)+\frac{t^{4}}{24} 8795(14)+\mathcal{O}\left(t^{5}\right),
\end{aligned}
$$

and for an initial gluon

$$
\begin{array}{r}
\langle\Delta z\rangle_{g}^{\mathrm{filt}, 3}=\frac{t^{3}}{6}\left(-36.657(8) C_{A}^{3}-5.3268(2) C_{A}^{2} n_{f} T_{R}-3.48461(7) C_{A} C_{F} n_{f} T_{R}\right. \\
\left.-3.5362(3) C_{F}^{2} n_{f} T_{R}-0.1526421(5) C_{A} n_{f}^{2} T_{R}^{2}-0.1021532(8) C_{F} n_{f}^{2} T_{R}^{2}\right) \\
+\frac{t^{4}}{24} 79258(213)+\mathcal{O}\left(t^{5}\right)
\end{array}
$$

where for the $t^{4}$ terms we have not extracted the explicit colour separation.

The all-order results for $\langle\Delta z\rangle^{\text {filt }}$ are given in figure 7 for $n_{\text {filt }}=2$ (upper row) and $n_{\text {filt }}=3$ (lower row). On one hand one observes how stable the filtered momentum is relative to the momentum of a single microjet. Taking $t=0.1$, which corresponds to $R_{(\text {filt })} \simeq 0.13$, an $n=2$ filtered jet retains $90 \%$ of the parton's momentum (gluon case), while an $n=3$ filtered jet retains nearly $95 \%$ of the momentum. This is to be compared to just $75 \%$ for a single microjet. This is in part a consequence of the fact that $\langle\Delta z\rangle^{\text {filt,n }}$ is non-zero only starting from order $t^{n}$ rather than order $t$. Interestingly, however, the convergence of the series in $t$ seems to be far worse. Considering the gluonic $n=3$ case, for $R_{\text {filt }}=0.2$ (a not unusual choice), the zeroth order approximation is closer to the full resummed result than any of the non-zero fixed-order results. We are not sure why this is the case, but it suggests that resummation effects should certainly be studied further if one is to carry out precision physics with filtered jets beyond their originally intended application of jet-mass determination in boosted-object taggers.

\subsubsection{Trimming}

In trimming one takes a jet of size $R_{0}$, reclusters its constituents on a smaller angular scale $R_{\text {trim }}<R_{0}$ and keeps (and merges) just the subjets with $p_{t}^{\text {subjet }} \geq f_{\text {cut }} p_{t}^{\text {jet }}$. As was the case for filtering, $t$ is now defined in terms of $R_{\text {trim }}$ rather than $R$.

The energy difference between the trimmed jet and the initial parton of flavour $i$ can then be expressed as a function of $f_{\text {cut }}$ by the equation

$$
\left\langle\Delta z\left(f_{\text {cut }}\right)\right\rangle_{i}^{\text {trim }}=\left[\sum_{j} \int_{f_{\text {cut }}}^{1} d z z f_{j / i}^{\text {incl }}(z, t)\right]-1=\sum_{j} \int_{0}^{f_{\text {cut }}} d z(-z) f_{j / i}^{\text {incl }}(z, t) .
$$

Given that the integral in the rightmost expression ranges only from $z=0$ to $f_{\text {cut }}$, it is 

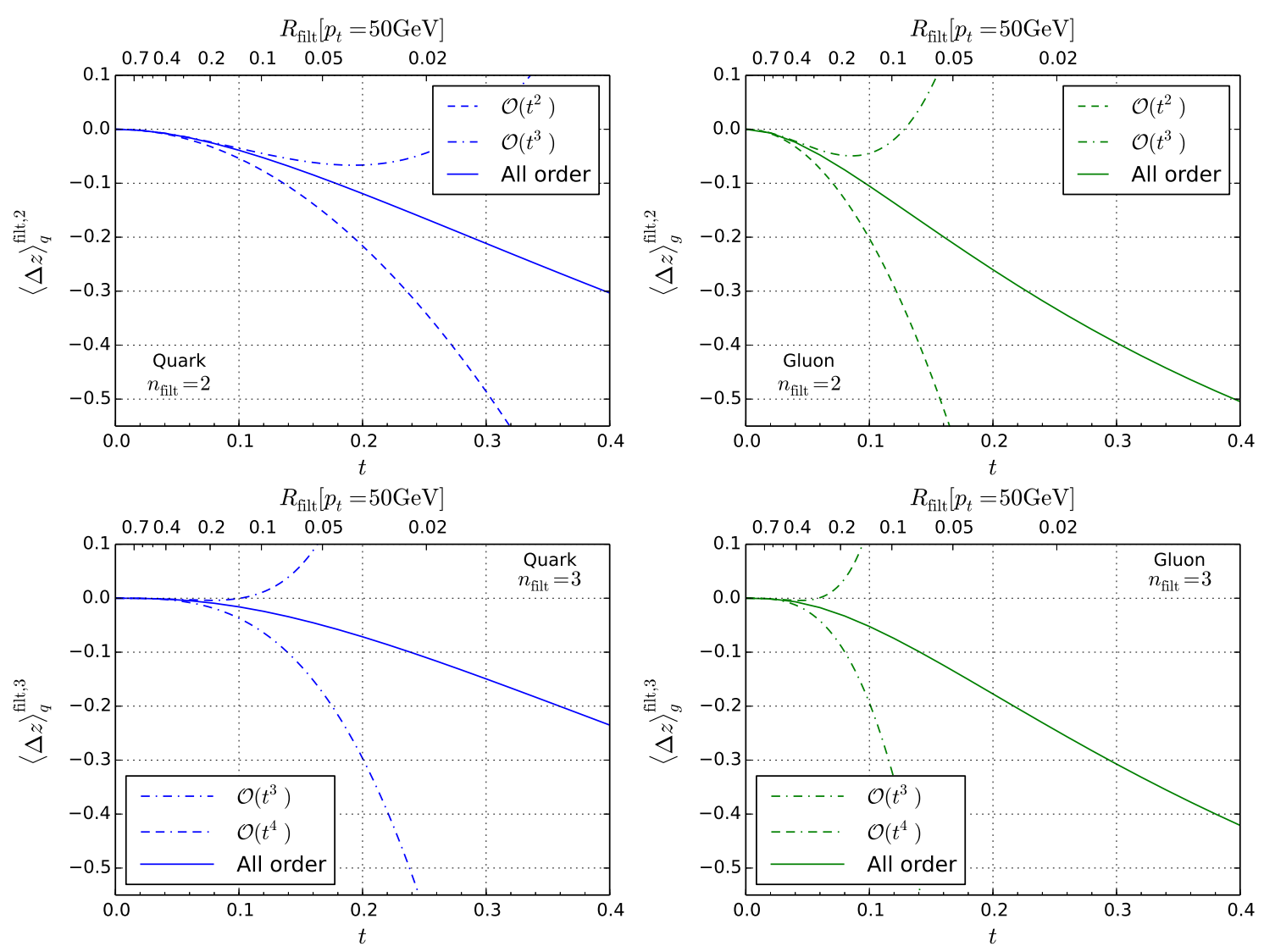

Figure 7. Average fractional jet energy loss $\Delta z$ after filtering with $n_{\text {filt }}=2$ (upper row) and $n_{\text {filt }}=3$ (lower row), as a function of $t$, for quark-induced jets (left) and gluon-induced jets (right). Resummed results are represented as solid lines. The second, third and fourth orders in $t$ are represented as dashed, dash-dotted and dash-dash-dotted lines respectively.

straightforward to see the that the result will be of order $f_{\text {cut }}$. For the quark case we have

$$
\begin{aligned}
\left\langle\Delta z\left(f_{\text {cut }}\right)\right\rangle_{q}^{\text {trim }}= & \frac{t}{2} C_{F}\left[3 f_{\text {cut }}^{2}+4 \ln \left(1-f_{\text {cut }}\right)\right]+ \\
+\frac{t^{2}}{2}\left[C _ { F } ^ { 2 } f _ { \text { cut } } ^ { 2 } \left(\frac{3}{4}-\right.\right. & \left.\frac{1}{2} \ln f_{\text {cut }}\right)+C_{A} C_{F} f_{\text {cut }}\left(4 \ln f_{\text {cut }}+\frac{8}{3}\right)+C_{A} C_{F} f_{\text {cut }}^{2}\left(2 \ln f_{\text {cut }}-\frac{7}{6}\right) \\
& \left.\quad-\frac{4}{3} n_{f} C_{F} T_{R} f_{\text {cut }}+n_{f} C_{F} T_{R} f_{\text {cut }}^{2}\left(-2 \ln f_{\text {cut }}-\frac{2}{3}\right)+\mathcal{O}\left(f_{\text {cut }}^{3}\right)\right], \quad
\end{aligned}
$$

where at order $t^{2}$ we have given just the first couple of terms in a series expansion in $f_{\text {cut }}$ (see appendix A.5 for the full expressions). For the gluon case we find

$$
\begin{aligned}
& \left\langle\Delta z\left(f_{\text {cut }}\right)\right\rangle_{g}^{\text {trim }}= \\
& \quad \frac{t}{6}\left[C_{A} f_{\text {cut }}^{2}\left(12-4 f_{\text {cut }}+3 f_{\text {cut }}^{2}\right)-2 T_{R} n_{f} f_{\text {cut }}^{2}\left(3-4 f_{\text {cut }}+3 f_{\text {cut }}^{2}\right)+12 C_{A} \ln \left(1-f_{\text {cut }}\right)\right]+ \\
& +\frac{t^{2}}{2}\left[C_{A}^{2} f_{\text {cut }}\left(4 \ln f_{\text {cut }}+\frac{10}{3}\right)+C_{A}^{2} f_{\text {cut }}^{2}\left(2 \ln f_{\text {cut }}+\frac{2}{3}\right)-n_{f} C_{A} T_{R} f_{\text {cut }}^{2}\left(\frac{19}{6}+2 \ln f_{\text {cut }}\right)\right. \\
& \left.\quad-\frac{8}{3} n_{f} C_{F} T_{R} f_{\text {cut }}-n_{f} C_{F} T_{R} f_{\text {cut }}^{2} \ln f_{\text {cut }}+\frac{2}{3} f_{\text {cut }}^{2} n_{f}^{2} T_{R}^{2}+\mathcal{O}\left(f_{\text {cut }}^{3}\right)\right]
\end{aligned}
$$


A key feature of the above equations is the presence at order $t^{2}$ of $f_{\text {cut }} \ln f_{\text {cut }}$ terms in the $C_{F} C_{A}$ and $C_{A}^{2}$ colour channels for quarks and gluons respectively. This should not be surprising, since trimming has two small parameters, $R_{\text {trim }}$ and $f_{\text {cut }}$, and to obtain a robust prediction it is advisable to resum the logarithms that arise from each of these small parameters. Terms $\alpha_{s}^{n} f_{\text {cut }} \ln ^{n} R^{2} \ln ^{n-1} f_{\text {cut }}$ are in fact implicitly included in our current approach, but ideally one would aim to resum also all the terms that appear without $\ln R^{2}$ enhancements as well, e.g. $\alpha_{s}^{n} f_{\text {cut }} \ln ^{n-1} f_{\text {cut }}$. The logarithms of $f_{\text {cut }}$ involve, for example, running-coupling effects beyond those considered here, non-global logarithms [64], and potentially also clustering logarithms [65]. We leave their study to future work. Without them, it seems difficult to use just the $\ln R^{2}$ resummation to draw conclusions about the convergence and size of trimming effects. We therefore give our full $\ln R^{2}$-resummed results for trimming only in appendix A.5, together with the complete analytical expressions for the $t^{2}$ terms and numerical determinations of the $t^{3}$ and $t^{4}$ contributions.

\section{Conclusions}

In this article we have introduced a method to resum terms $\left(\alpha_{s} \ln R^{2}\right)^{n}$ to all orders and applied it to a wide range of jet observables. One key observation is that small- $R$ effects can be substantial, for example reducing the inclusive-jet spectrum by $30-50 \%$ for gluon jets when $R$ is in the range $0.4-0.2$.

The set of observables we considered should not be seen as exhaustive: for example we might equally well have considered quantities such as dijet asymmetries, microjet multiplicities or, with a little further work, jet shapes. The program that we used to obtain the results presented here can be applied also to a number of these other observables, and will be made public in due course to facilitate such studies.

One should not forget that the leading logarithms of $R$ that we resummed are also included in parton-shower Monte Carlo programs that include angular ordering, whether directly, or through some other implementation of colour coherence. However it is nontrivial to understand their role separately from the many other physical effects present in Monte Carlo generators and it is also difficult to merge them with other logarithmic resummations or fixed-order calculations, especially beyond NLO. With the resummations in the form that we have given here, one can directly isolate the small- $R$ effects and merging with other calculations becomes straightforward, as we illustrated briefly for jet veto resummations. We also saw that the resummation made it possible to estimate the range of validity in $R$ of fixed-order perturbative calculations. One potential area of application, where $R$ is genuinely small, is in substructure studies, where we saw for filtering that the resummation effects are substantial, and fixed-order convergence intriguingly poor.

We have not considered the detailed phenomenology of small- $R$ effects in this paper, leaving such a study instead to future work [47]. This study should, among other things, also consider the role of power suppressed hadronisation corrections proportional to $\Lambda_{\mathrm{QCD}} / p_{t}$, which are known to receive a $1 / R$ enhancement at small $R[22,66,67]$. Specific observables that could be examined are inclusive jet spectra, jet-veto efficiencies in Higgs production and jet $p_{t}$ imbalances in heavy-ion collisions. Other future work might consider 
small- $R$ logarithms in conjunction also with threshold resummation. Finally it would be of interest to understand how to go beyond LL accuracy for small- $R$ resummations. We believe that the techniques that apply would be of relevance also for the resummation of jet substructure observables such as the modified Mass Drop Tagger's jet-mass distribution beyond the LL accuracy obtained so far [68].

\section{Acknowledgments}

We are grateful to Matteo Cacciari for collaboration in the early stages of this work and numerous discussions throughout, to Andrea Banfi, Pier Monni and Giulia Zanderighi for helpful suggestions, to Simone Alioli and Jon Walsh for exchanges about their calculation of the jet veto efficiency and to University College London for hospitality while this work was being completed. This work was supported by the European Commission under ITN grant LHCPhenoNet, PITN-GA-2010-264564, by ERC advanced grant Higgs@LHC, by the U.K.'s STFC, by the French Agence Nationale de la Recherche, under grant ANR10-CEXC-009-01, and by the ILP LABEX (ANR-10-LABX-63) supported by French state funds managed by the ANR within the Investissements d'Avenir programme under reference ANR-11-IDEX-0004-02.

\section{A Analytical expressions}

For all the second order coefficients given numerically in the main text, this appendix provides the full analytical expressions. It also gives analytical and numerical results for some quantities that for brevity were left out of the main text.

The results frequently involve the polylogarithm $\mathrm{Li}_{s}(z)$, defined in the unit circle by

$$
\operatorname{Li}_{s}(z)=\sum_{k=1}^{\infty} \frac{z^{k}}{k^{s}} \quad s \in \mathbb{N}, \quad|z|<1,
$$

and by analytic continuation for $|z|>1$. The polylogarithm also follows the recursive relation

$$
\operatorname{Li}_{1}(z)=-\ln (1-z), \quad \operatorname{Li}_{s+1}(z)=\int_{0}^{z} \frac{\mathrm{Li}_{s}(t)}{t} d t
$$

\section{A.1 Hardest microjet $\langle\Delta z\rangle$}

Let us first consider the energy difference between the the hardest subjet and the initiating parton, as expressed in eq. (4.8). For the case of an initiating quark, given by eq. (4.9), 
the $c_{2}$ coefficient of $\langle\Delta z\rangle^{\text {hardest }}$ is

$$
\begin{aligned}
c_{2}\left[\langle\Delta z\rangle_{q}^{\text {hardest }}\right]= & C_{F}^{2}\left[\frac{97}{144}-\pi^{2}+\frac{5 \ln 2}{12}-2 \ln ^{2} 2+4 \ln ^{2} 3+8 \operatorname{Li}_{2}\left(\frac{2}{3}\right)\right]+ \\
& +2 C_{F} T_{R} n_{f}\left[\frac{41}{12}+12 \ln 3-24 \ln 2\right]+ \\
+ & C_{F} C_{A}\left[-\frac{967}{144}+\frac{\pi^{2}}{3}-4 \operatorname{Li}_{2}\left(-\frac{1}{3}\right)-4 \operatorname{Li}_{2}\left(\frac{2}{3}\right)-2 \ln ^{2} 2-\right. \\
& \left.-4 \ln ^{2} 3+\frac{553}{12} \ln 2-\frac{99}{4} \ln 3+8 \ln 2 \ln 3\right],
\end{aligned}
$$

and when the initiating parton is a gluon, corresponding to eq. (4.10), we have

$$
\begin{aligned}
c_{2}\left[\langle\Delta z\rangle_{g}^{\text {hardest }}\right]=C_{A} T_{R} n_{f}\left[\frac{3527}{432}-\frac{176}{3} \ln 2+\frac{727}{24} \ln 3\right]+\frac{7}{72} n_{f}^{2} T_{R}^{2}+ & \\
& +\frac{1}{432} C_{F} T_{R} n_{f}[451-3420 \ln 2+1548 \ln 3]+ \\
+C_{A}^{2}\left[\frac{2 \pi^{2}}{3}-\right. & \frac{2089}{288}-4 \ln ^{2} 2-2 \ln ^{2} 3+\ln 2\left(\frac{1339}{24}+8 \ln 3\right)-\frac{475}{16} \ln 3- \\
& \left.-4 \operatorname{Li}_{2}\left(\frac{2}{3}\right)+4 \operatorname{Li}_{2}\left(\frac{1}{4}\right)-4 \operatorname{Li}_{2}\left(\frac{3}{4}\right)\right] .
\end{aligned}
$$

\section{A.2 Logarithmic moment $\langle\ln z\rangle$}

Here we give our full set of results for the logarithmic moment of $f^{\text {hardest }}$ as expressed in eq. (4.16). Results for an initiating quark were not given in detail in the main text. The coefficients $c_{1 \ldots 3}$ of $t, t^{2} / 2$ and $t^{3} / 6$ are respectively

$$
c_{1}\left[\langle\ln z\rangle_{q}^{\text {hardest }}\right]=\frac{C_{F}}{6}\left(9-\pi^{2}-9 \ln 2\right),
$$




$$
\begin{gathered}
c_{2}\left[\langle\ln z\rangle_{q}^{\text {hardest }}\right]=\frac{1}{432} C_{F} C_{A}\left[-3887+786 \ln \left(\frac{9}{4}\right)-24 \pi^{2}\left(-92+54 \ln 2-30 \ln 3+\ln \left(\frac{729}{64}\right)\right)-\right. \\
-144(91+72 \operatorname{arccoth}(5)) \operatorname{Li}_{2}\left(\frac{1}{3}\right)-13392 \operatorname{Li}_{2}\left(\frac{2}{3}\right)- \\
-288 \ln ^{2} 2(5+3 \ln 729)-12 \ln 3\left(1175+24 \ln 9(23+\ln 27)-72 \operatorname{Li}_{2}\left(\frac{1}{9}\right)\right)+ \\
+24 \ln 2\left(1166-33 \ln \left(\frac{9}{4}\right)+36 \ln 3(17+7 \ln 3)+72 \operatorname{Li}_{2}\left(-\frac{1}{2}\right)+144 \operatorname{Li}_{2}\left(\frac{2}{3}\right)\right)+ \\
\left.+288 \ln ^{3} 2+3456 \operatorname{Li}_{3}\left(-\frac{1}{2}\right)+1728 \operatorname{Li}_{3}\left(-\frac{1}{3}\right)+3456 \operatorname{Li}_{3}\left(\frac{2}{3}\right)-432 \zeta(3)\right]+ \\
+\frac{1}{216} C_{F} T_{R} n_{f}\left[1457-24 \pi^{2}+36 \ln 3(135+\ln 81)-12 \ln 2(833+\ln 4096)+288 \operatorname{Li}_{2}\left(\frac{2}{3}\right)\right]+ \\
+\frac{1}{24} C_{F}^{2}\left[-42 \operatorname{Li}_{2}\left(-\frac{1}{2}\right)-126 \operatorname{Li}_{2}\left(\frac{1}{3}\right)-30 \operatorname{Li}_{2}\left(\frac{3}{4}\right)-96 \operatorname{Li}_{3}\left(-\frac{1}{2}\right)+192 \operatorname{Li}_{3}\left(\frac{1}{3}\right)-96 \operatorname{Li}_{3}\left(\frac{2}{3}\right)-\right. \\
-24 \operatorname{Li}_{3}\left(\frac{1}{9}\right)-\ln 3\left(192 \operatorname{Li}_{2}\left(\frac{2}{3}\right)+96 \operatorname{Li}_{2}\left(-\frac{1}{3}\right)-102+128 \ln 3+63 \ln 3\right)+ \\
+6 \ln 2\left(-16 \operatorname{Li}_{2}\left(\frac{1}{3}\right)+16 \operatorname{Li}_{2}\left(\frac{2}{3}\right)+16 \operatorname{Li}_{2}\left(\frac{3}{4}\right)-41+\ln 3(31+24 \ln 3)\right)- \\
\left.\quad-60 \zeta(3)+9+208 \ln ^{3} 2-3(39+32 \ln 3) \ln 22+2 \pi^{2}(3+8 \ln 3)\right] \\
\simeq-0.73199 C_{A} C_{F}+1.0414 C_{F}^{2}-0.114427 C_{F} n_{f} T_{R}, \quad(\mathrm{~A} .5 \mathrm{~b})
\end{gathered}
$$

and

$$
\begin{aligned}
& c_{3}\left[\langle\ln z\rangle_{q}^{\text {hardest }}\right]=-0.17394(5) C_{F}^{3}+0.66631(2) C_{A}^{2} C_{F}-0.81869(4) C_{A} C_{F}^{2}+ \\
& \quad+0.595241(9) C_{A} C_{F} n_{f} T_{R}-0.534856(8) C_{F}^{2} n_{f} T_{R}+0.076288(2) C_{F} n_{f}^{2} T_{R}^{2}
\end{aligned}
$$

In the gluon case, expressed in eq. (4.17), the analytic form for the second order coefficient is

$$
\begin{gathered}
c_{2}\left[\langle\ln z\rangle_{g}^{\text {hardest }}\right]=C_{A} T_{R} n_{f} \frac{1}{216}\left[1697+24 \pi^{2}+6336 \ln 3-36 \ln 2(347+4 \ln 2)\right]+ \\
+C_{F} T_{R} n_{f} \frac{1}{36}\left[97+8 \pi^{2}+48 \ln ^{2} 2-48 \ln ^{2} 3+24 \ln \left(\frac{3}{2}\right)+36 \ln \left(\frac{2187}{32768}\right)-96 \operatorname{Li}_{2}\left(\frac{2}{3}\right)\right]- \\
-\frac{1}{144} C_{A}^{2}\left[3624 \operatorname{Li}_{2}\left(\frac{2}{3}\right)+8 \ln 2\left(108 \operatorname{Li}_{2}\left(\frac{2}{3}\right)-1163+6 \ln 3(9 \ln 3-22)\right)+\right. \\
+12 \ln 3\left(48 \operatorname{Li}_{2}\left(-\frac{1}{3}\right)+384+\ln 3(151+16 \ln 3)\right)+36 \operatorname{Li}_{2}\left(\frac{3}{4}\right)\left(65-4 \ln \left(\frac{81}{8}\right)\right)- \\
-72 \zeta(3)+1531+2208 \ln ^{3} 2+192 \ln ^{3}\left(\frac{4}{3}\right)-2304 \ln ^{2} 2(1+\ln (3))+288 \ln 3 \ln ^{2}\left(\frac{4}{3}\right)+ \\
\left.+24 \pi^{2}\left(\ln \left(\frac{81}{64}\right)-27\right)+12 \ln 4 \ln \left(\frac{4}{3}\right)(151-60 \ln 2)\right] \\
+\frac{1}{54} n_{f}^{2} T_{R}^{2}(23-24 \ln 2) .
\end{gathered}
$$




\section{A.3 Jet flavour}

The analytical form of the second-order coefficient for the probability for an initiating quark to change flavour to a gluon microjet, expressed numerically in eq. (4.19), is

$$
\begin{aligned}
& c_{2}[\mathcal{P}(g \mid q)]=-\frac{1}{12} C_{F} n_{f} T_{R}[16 \ln 2-5]- \\
&-\frac{1}{48} C_{F}^{2}\left[-192 \operatorname{Li}_{2}\left(\frac{3}{4}\right)+16 \pi^{2}+49+12 \ln 2(9-40 \ln 2)+48 \ln 3(\ln 256-3)\right]+ \\
&+\frac{1}{144} C_{F} C_{A}\left[288 \operatorname{Li}_{2}\left(\frac{3}{4}\right)-\right. 48 \pi^{2}+1463+5076 \ln 3+ \\
&+36 \ln 2(-277+24 \ln 2-16 \ln 3)] .
\end{aligned}
$$

For the probability of a change of flavour from an initiating gluon to a quark microjet jet, corresponding to eq. (4.20), we have

$$
\begin{aligned}
c_{2}[\mathcal{P}(q \mid g)]= & -\frac{1}{27} C_{A} n_{f} T_{R}[455-2988 \ln 2+1476 \ln 3]-\frac{12}{27} n_{f}^{2} T_{R}^{2}- \\
& -\frac{1}{27} C_{F} n_{f} T_{R}\left[288 \ln \left(\frac{4}{3}\right)-71\right] .
\end{aligned}
$$

\section{A.4 Filtering}

We give next the analytical form of the $c_{2}$ coefficient for the total energy loss when taking the sum of the 2 hardest microjets. For an initial quark, corresponding to eq. (4.22), this is

$$
\begin{aligned}
c_{2}\left[\langle\Delta z\rangle_{q}^{\text {filt, }, 2}\right]= & \frac{1}{18} C_{F}^{2}\left[72 \operatorname{Li}_{2}\left(\frac{3}{4}\right)-12 \pi^{2}\right. \\
+\frac{2}{9} C_{F} T_{R} n_{f}[43-216 \operatorname{arccoth}(5)]+ & \frac{1}{12} C_{A} C_{F}\left[48 \operatorname{Li}_{2}\left(\frac{2}{3}\right)+48 \operatorname{Li}_{2}\left(-\frac{1}{3}\right)-4 \pi^{2}-111-\right. \\
& -96 \ln 2(3+\ln 3)+3 \ln 3(99+16 \ln 3)],
\end{aligned}
$$

while for the gluon case, corresponding to eq. (4.23), we have

$$
\begin{aligned}
c_{2}\left[\langle\Delta z\rangle_{g}^{\mathrm{filt}, 2}\right]=\frac{1}{216} C_{A} & T_{R} n_{f}[2588+6480 \ln 2-6543 \ln 3]+ \\
& +\frac{1}{108} C_{F} T_{R} n_{f}[146+324 \ln 2-387 \ln 3]- \\
-C_{A}^{2} & \frac{1}{48}\left[96 \operatorname{Li}_{2}\left(\frac{1}{3}\right)-96 \operatorname{Li}_{2}\left(\frac{2}{3}\right)+96 \operatorname{Li}_{2}\left(\frac{1}{4}\right)-288 \operatorname{Li}_{2}\left(\frac{3}{4}\right)+\right. \\
& \left.+48 \pi^{2}+500-1425 \ln 3+48 \ln 2(27-8 \ln 2+10 \ln 3)\right] .
\end{aligned}
$$




\section{A.5 Trimming}

The full second order results for $\langle\Delta z\rangle^{\text {trim }}$ are

$$
\begin{aligned}
& c_{2}\left[\langle\Delta z\rangle_{q}^{\text {trim }}\right]=\frac{1}{12} C_{F}^{2}\left[-30 f_{\text {cut }}^{2} \ln f_{\text {cut }}+16 \ln \left(1-f_{\text {cut }}\right)\left(3 f_{\text {cut }}^{2}+3 \ln \left(1-f_{\text {cut }}\right)-2\right)+\right. \\
& \left.+48 \mathrm{Li}_{2}\left(1-f_{\text {cut }}\right)+16\left(f_{\text {cut }}^{2}+6\right) f_{\text {cut }} \operatorname{arctanh}\left(1-2 f_{\text {cut }}\right)+6 f_{\text {cut }}^{3}+5 f_{\text {cut }}^{2}+16 f_{\text {cut }}-8 \pi^{2}\right]+ \\
& +\frac{1}{6} C_{A} C_{F}\left[\left(f_{\text {cut }}-1\right)\left(-\left(2 f_{\text {cut }}^{3}+9 f_{\text {cut }}^{2}+32 f_{\text {cut }}\right)-4\left(\left(f_{\text {cut }}-2\right) f_{\text {cut }}+4\right) \ln \left(1-f_{\text {cut }}\right)\right)+\right. \\
& \left.+4 f_{\text {cut }}\left(2 f_{\text {cut }}^{2}+3 f_{\text {cut }}+6\right) \ln f_{\text {cut }}\right]+ \\
& +\frac{2}{3} C_{F} T_{R} n_{f} f_{\text {cut }}\left[\left(f_{\text {cut }}-1\right)\left(f_{\text {cut }}+1\right)\left(f_{\text {cut }}+2\right)-f_{\text {cut }}\left(2 f_{\text {cut }}+3\right) \ln f_{\text {cut }}\right] \text {, }
\end{aligned}
$$

for the quark case, and

$$
\begin{gathered}
c_{2}\left[\langle\Delta z\rangle_{g}^{\text {trim }}\right]=\frac{1}{36} C_{A}^{2}\left[-36\left(f_{\text {cut }}-4\right) f_{\text {cut }}^{3} \ln f_{\text {cut }}+24 \ln \left(1-f_{\text {cut }}\right)\left(\left(3 f_{\text {cut }}^{2}-4 f_{\text {cut }}+12\right) f_{\text {cut }}^{2}+\right.\right. \\
\left.\left.+6 \ln \left(1-f_{\text {cut }}\right)\right)+144 \mathrm{Li}_{2}\left(1-f_{\text {cut }}\right)-75 f_{\text {cut }}^{4}+16 f_{\text {cut }}^{3}-84 f_{\text {cut }}^{2}+264 f_{\text {cut }}-24 \pi^{2}\right]- \\
-\frac{1}{18} C_{A} T_{R} n_{f}\left[12\left(3 f_{\text {cut }}^{4}-4 f_{\text {cut }}^{3}+3 f_{\text {cut }}^{2}+2\right) \ln \left(1-f_{\text {cut }}\right)+12 f_{\text {cut }}^{2}\left(8 f_{\text {cut }}+3\right) \ln f_{\text {cut }}-\right. \\
\left.\quad-57 f_{\text {cut }}^{4}+8 f_{\text {cut }}^{3}+69 f_{\text {cut }}^{2}+24 f_{\text {cut }}\right]+ \\
+\frac{1}{3} C_{F} T_{R} n_{f}\left[\left(4-6 f_{\text {cut }}^{2}\right) \ln \left(1-f_{\text {cut }}\right)+4\left(4 f_{\text {cut }}^{3}-3 f_{\text {cut }}^{4}\right) \operatorname{arctanh}\left(1-2 f_{\text {cut }}\right)-\right. \\
\left.-3 f_{\text {cut }}^{2} \ln f_{\text {cut }}+2 f_{\text {cut }}^{4}+2 f_{\text {cut }}^{2}-4 f_{\text {cut }}\right]+\frac{2}{9} T_{R}^{2} n_{f}^{2} f_{\text {cut }}^{2}\left(3 f_{\text {cut }}^{2}-4 f_{\text {cut }}+3\right), \quad \text { (A.12) }
\end{gathered}
$$

for the gluon case. Numerical results for the third order terms are given in figures 8 .

All-order results for the widely used choice of $f_{\text {cut }}=0.05$ are shown in figure 9 as a function of $t$. As with filtering, the energy loss from trimmed jets with a given $R_{\text {trim }}$ is much reduced relative to that from a single microjet with that same radius $R_{\text {trim }}$. With this specific $f_{\text {cut }}$ value, the energy loss is somewhat smaller even than for filtering. Additionally the convergence of the series appear to be far better. However, as was discussed in section 4.3.2, one should keep in mind that trimming also leads to logarithms of $f_{\text {cut }}$, only some of which are included in our resummation. Their potential importance can be appreciated by considering that the full calculation would involve an integral over $\alpha_{s}$ down to scale $f_{\text {cut }} R_{\text {trim }} p_{t}$, which is much smaller than the scale $R_{\text {trim }} p_{t}$ that is included in our small- $R$ resummation. Indeed for the choice of $p_{t}=50 \mathrm{GeV}$ and $f_{\text {cut }}=0.05$ that we use in figure 9 , any $R_{\text {trim }}<0.4$ would force us to consider scales below $1 \mathrm{GeV}$. 

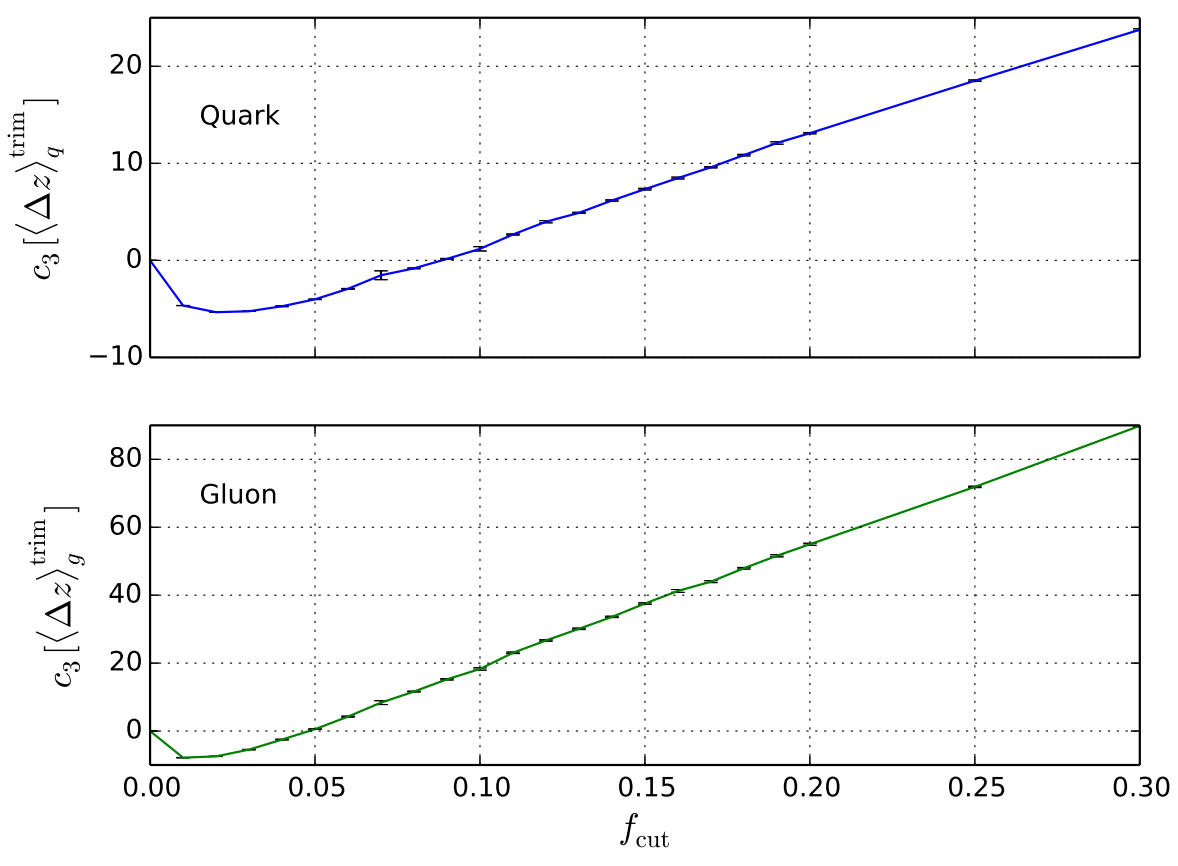

Figure 8. The third order coefficients $c_{3}\left(\langle\Delta z\rangle^{\text {trim }}\right)$, as a function of $f_{\text {cut }}$, for quark (top) and gluoninduced (bottom) jets. The solid lines are simply intended to guide the eye and do not provide any information beyond what is specified by the points.
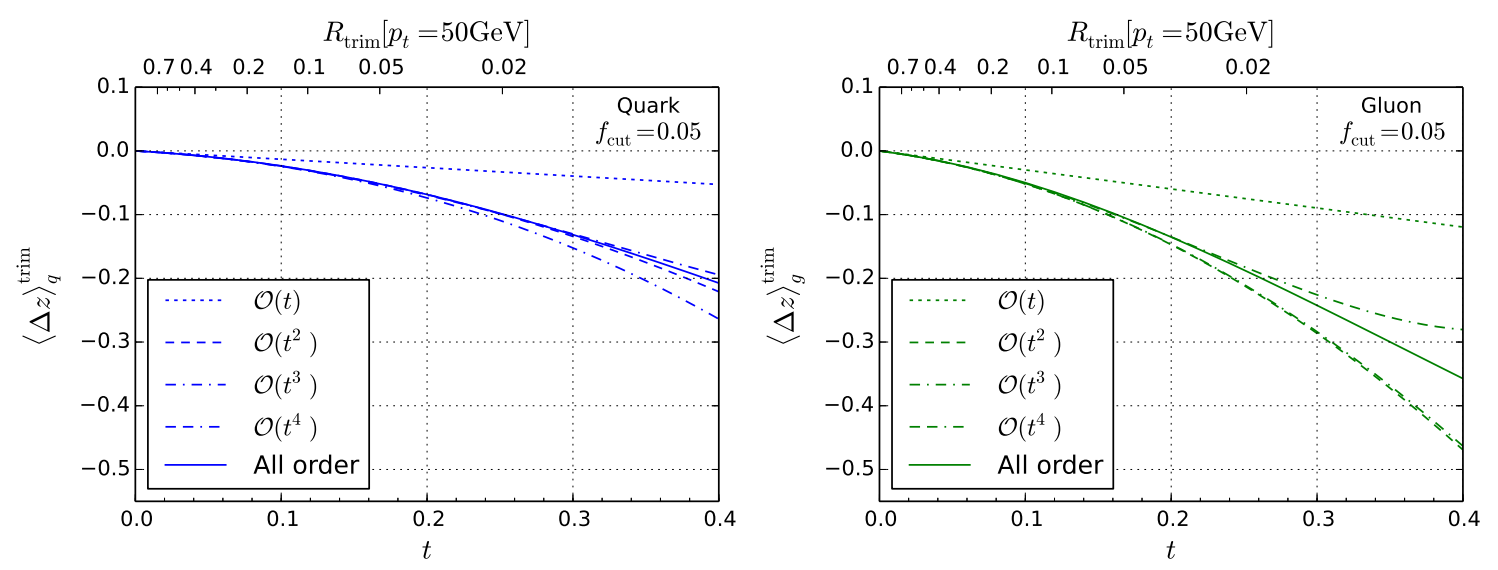

Figure 9. Average fractional jet energy loss $\Delta z$ after trimming with $f_{\text {cut }}=0.05$ as a function of $t$ for quarks (left) and gluons (right), with $\ln R_{\text {trim }}^{2}$ resummation, but not $\ln f_{\text {cut }}$ resummation. 


\section{B Comparisons and fixed-order cross checks}

\section{B.1 Comparison with the literature}

A numerical calculation of the $\alpha_{s}^{3} \ln \frac{Q}{p_{t}} \ln ^{2} R^{2}$ contribution to the jet veto efficiency in the case of $g g \rightarrow H$ production was given recently by Alioli and Walsh (AW) [25]. This section provides the detailed comparison.

First let us rewrite the first two orders of eq. (4.17) as an expansion in powers of $\alpha_{s}$, taking into account eq. (3.2) for the conversion between the $t$ and $\alpha_{s}$ expansions:

$$
\begin{array}{r}
\langle\ln z\rangle_{g}^{\text {hardest }}=\frac{\alpha_{s}\left(p_{t}\right)}{2 \pi} \ln \frac{1}{R^{2}}\left[\frac{1}{72} C_{A}\left(131-12 \pi^{2}-132 \ln 2\right)+\frac{1}{36} n_{f} T_{R}(-23+24 \ln 2)\right] \\
+\frac{\alpha_{s}^{2}\left(p_{t}\right)}{4 \pi^{2}} \ln ^{2} \frac{1}{R^{2}}\left[-0.36982 C_{F} n_{f} T_{R}+0.117861 n_{f}^{2} T_{R}^{2}+0.589237 C_{A} n_{f} T_{R}-0.901568 C_{A}^{2}\right]+ \\
+\mathcal{O}\left(\alpha_{s}^{3}\right), \quad(\text { B. } 1)
\end{array}
$$

where $p_{t}$ is the transverse-momentum above which jets are vetoed. AW have an expansion for the $R$-dependent terms in the jet-veto probability, eqs. (1.1) and (1.3) of ref. [25], whose leading logarithms of $R$ read

$$
\exp \left[C_{n}^{(n-1)}\left(\frac{\alpha_{s}\left(p_{t}\right) C_{A}}{\pi}\right)^{n} \ln \frac{Q}{p_{t}} \ln ^{n-1} R^{2}\right] .
$$

Substituting eq. (B.1) into eq. (4.15) gives our result for $C_{3}^{(2)}$,

$$
C_{3}^{(2)}=\left(0.36982 \frac{C_{F} n_{f} T_{R}}{C_{A}^{2}}-0.589237 \frac{n_{f} T_{R}}{C_{A}}-0.117861 \frac{n_{f}^{2} T_{R}^{2}}{C_{A}^{2}}+0.901568\right) \simeq 0.46566 .
$$

The results from AW that can be straightforwardly compared are the $C_{F} n_{f} T_{R}$ term and the full result. For the $C_{F} n_{f} T_{R}$ term, including the explicit colour factors, our result is $0.36982 \frac{C_{F} n_{f} T_{R}}{C_{A}^{2}} \simeq 0.1370$ (for $n_{f}=5$ ), with which AW's result of $0.1405 \pm 0.0011$ is marginally compatible. For the full result, our coefficient of $C_{3}^{(2)} \simeq 0.46566$ is to be compared with that from $\mathrm{AW}$ of $C_{3}^{(2)}=-0.356 \pm 0.011$. After consulting with the authors, they identified the origin of the difference as being due to the fact that they effectively did not include the running coupling corrections that appear at order $\alpha_{s}^{2}$ in the expansion of $t$. Once this is resolved, their answers come into reasonable agreement with ours.

\section{B.2 Fixed-order cross checks}

On one hand the results we have presented are sufficiently straightforward that there should be no need for cross-checks from full fixed-order calculations. However, given the initial disagreement with the results of [25], such cross-checks became desirable. In principle one could take a program such as MCFM and directly take the limit of small $p_{t}$ and small $R$ in the Higgs plus two jet process at NLO, in order to directly cross-check the $C_{3}^{(2)}$ coefficient in eq. (B.3). However in practice, we believe that it would be almost impossible to take the 
appropriate double limit, simultaneously satisfying the requirements of being sufficiently asymptotic and having sufficient Monte Carlo statistics.

Instead we have opted to test the basic framework and in particular the results for the distribution $f^{\text {hardest }}(z)$ integrated up to some finite $z$,

$$
P_{z<z_{\max }}=\int_{0}^{z_{\max }} d z f^{\text {hardest }}(z)
$$

which is closely related to (one minus) the probability of vetoing a jet. We examined the $e^{+} e^{-} \rightarrow 3$ jet process at next-to-leading order (NLO), using the Event2 program [56]. We initially clustered the events with the $e^{+} e^{-}$version of the inclusive Cambridge/Aachen algorithm [28, 29], "ee-genkt" with $p=0$ and $R_{0}=1.5$ as defined in FastJet [69]. Then, for each jet with an energy above $z_{\max } Q / 2$, where $Q$ is the centre-of-mass energy, we progressively unclustered the jet until we reached a configuration where each of the subjets had an energy of less than $z_{\max } Q / 2$. We identified the Cambridge/Aachen distance for that declustering,

$$
d^{(\mathrm{C} / \mathrm{A})}=\frac{1-\cos \theta}{1-\cos R_{0}} \simeq \frac{\theta^{2}}{2\left(1-\cos R_{0}\right)},
$$

where $\theta$ is the angle between the two subjets or particles that got declustered, and the rightmost (approximate) expression holds for small $\theta$. We then added an entry for that event to the corresponding bin of $\ln 1 / d^{(\mathrm{C} / \mathrm{A})}$. This gives us a numerical result for

$$
\frac{d P_{z<z_{\max }}}{d \ln 1 / d^{(\mathrm{C} / \mathrm{A})}}=\frac{d}{d \ln 1 / d^{(\mathrm{C} / \mathrm{A})}} \int_{0}^{z_{\max }} d z f^{\text {hardest }}(z),
$$

because for values of $d^{(\mathrm{C} / \mathrm{A})}$ smaller than that of the bin, the hardest $z$ satisfies $z>z_{\max }$, while for values of $d^{(\mathrm{C} / \mathrm{A})}$ larger than that of the bin the hardest $z$ satisfies $z<z_{\max }$.

Up to order $\alpha_{s}^{2}$, we expect the result to be

$$
\begin{aligned}
& \frac{d P_{z<z_{\max }}}{d \ln 1 / d^{(\mathrm{C} / \mathrm{A})}}=\frac{\alpha_{s}}{2 \pi}\left[c_{1}\left[P_{z<z_{\max }}\right]+\mathcal{O}\left(d^{(\mathrm{C} / \mathrm{A})}\right)\right]+ \\
&+\left(\frac{\alpha_{s}}{2 \pi}\right)^{2}\left[\left(b_{0} c_{1}\left[P_{z<z_{\max }}\right]+c_{2}\left[P_{z<z_{\max }}\right]\right) \ln \frac{1}{d^{(\mathrm{C} / \mathrm{A})}}+\mathcal{O}(1)\right]+\mathcal{O}\left(\alpha_{s}^{3}\right)
\end{aligned}
$$

where the $c_{1}$ and $c_{2}$ coefficients are precisely those worked out in this paper at LL order in the small- $R$ limit (had we evaluated subleading terms, care would have been needed concerning the difference between $d^{(C / A)}$ and $R^{2}$, however since this just reduces to a constant factor for small angles, it is irrelevant at LL order). Figure 10 (left) shows the difference between the actual NLO term in Event2 (without the LO contribution) and the expected $\mathcal{O}\left(\alpha_{s}^{2}\right)$ term of eq. (B.7), as evaluated from the first and second order expansions of our small- $R$ resummation. It is shown as a function of $\ln 1 / d^{(\mathrm{C} / \mathrm{A})}$, for $z_{\max }=0.6$, both for the total result and separately for each of the colour-factor contributions. In all cases asymptotically it is flat, indicating that we have subtracted the correct $\mathcal{O}\left(\alpha_{s}^{2} \ln \frac{1}{d^{(\mathrm{C} / \mathrm{A})}}\right)$ contribution. The right-hand plot shows the expected results for the coefficients of the $\mathcal{O}\left(\alpha_{s}\right)$ and $\mathcal{O}\left(\alpha_{s}^{2}\right)$ LL terms, versus those observed in Event2, as a function of $z_{\text {max }}$. Again the agreement is very good. We could also have directly studied $\langle\ln z\rangle^{\text {hardest }}$, however 

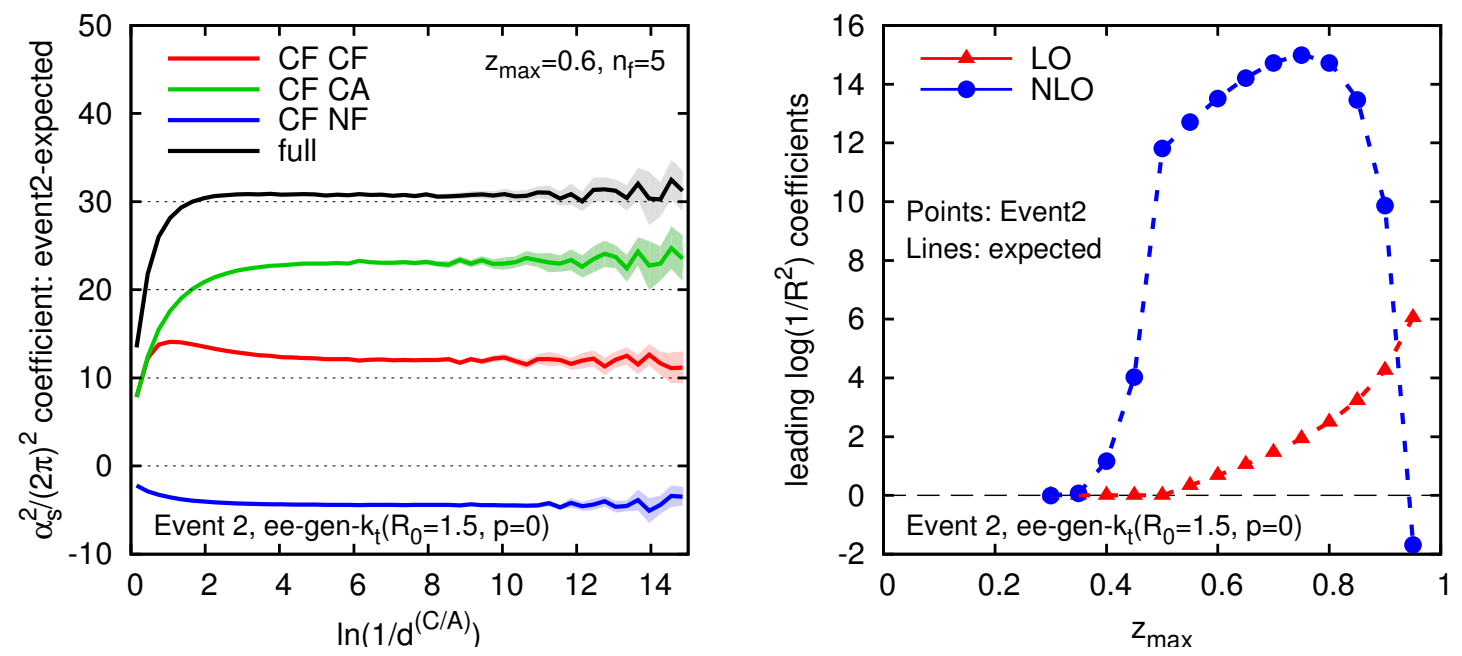

Figure 10. Left: difference between Event2 NLO results and the expected $\left.\alpha_{s}^{2} \ln 1 / d^{(\mathrm{C} / \mathrm{A}}\right)$ coefficient for the derivative with respect to $\ln 1 / d^{(\mathrm{C} / \mathrm{A})}$ of $P_{z<z_{\max }}$, for $z_{\max }=0.6$. The lines should be flat asymptotically if Event 2 and our calculations agree. Right: comparison of the expected and Event2 results for the $\left[\left(\alpha_{s} / 2 \pi\right) \ln 1 / R^{2}\right]^{n}$ coefficients for $P_{z<z_{\max }}$, as a function of $z_{\max }$, for $n=1$ (LO) and $n=2(\mathrm{NLO})$.

the test as shown in figure 10 is in some respects more complete because it probes the $f^{\text {hardest }}(z)$ distribution differentially. Two final remarks are in order. Firstly, these tests do not unequivocally demonstrate that it is the $\langle\ln z\rangle^{\text {hardest }}$ moment that is the relevant quantity for a jet-veto probability - for this one still relies on the calculation in eqs. (4.11)(4.15). Secondly, Event2 provides a test of our results only for quark jets. Nevertheless, the property of angular ordering that we rely on for our calculations is common to quark and to gluon jets.

Open Access. This article is distributed under the terms of the Creative Commons Attribution License (CC-BY 4.0), which permits any use, distribution and reproduction in any medium, provided the original author(s) and source are credited.

\section{References}

[1] G.F. Sterman and S. Weinberg, Jets from Quantum Chromodynamics, Phys. Rev. Lett. 39 (1977) 1436 [inSPIRE].

[2] S. Catani, Y.L. Dokshitzer, M.H. Seymour and B.R. Webber, Longitudinally invariant $K_{t}$ clustering algorithms for hadron hadron collisions, Nucl. Phys. B 406 (1993) 187 [InSPIRE].

[3] ATLAS collaboration, Jet energy measurement and its systematic uncertainty in proton-proton collisions at $\sqrt{s}=7 \mathrm{TeV}$ with the ATLAS detector, Eur. Phys. J. C 75 (2015) 17 [arXiv: 1406.0076] [INSPIRE].

[4] CMS collaboration, Determination of Jet Energy Calibration and Transverse Momentum Resolution in CMS, 2011 JINST 6 P11002 [arXiv:1107.4277] [INSPIRE]. 
[5] STAR collaboration, M. Ploskon, Inclusive cross section and correlations of fully reconstructed jets in $\sqrt{s_{N N}}=200-G E V$ Au+Au and $p+p$ collisions, Nucl. Phys. A 830 (2009) 255C [arXiv:0908.1799] [INSPIRE].

[6] PHENIX collaboration, Y.-S. Lai, Direct jet reconstruction in $p+p$ and $C u+C u$ at PHENIX, arXiv:0911.3399 [INSPIRE].

[7] ALICE collaboration, Measurement of charged jet suppression in $\mathrm{Pb}$-Pb collisions at $\sqrt{s_{N N}}$ = 2.76 TeV, JHEP 03 (2014) 013 [arXiv:1311.0633] [INSPIRE].

[8] ATLAS collaboration, Measurement of inclusive jet charged-particle fragmentation functions in $\mathrm{Pb}+\mathrm{Pb}$ collisions at $\sqrt{s_{N N}}=2.76$ TeV with the ATLAS detector, Phys. Lett. $\mathbf{B} 739$ (2014) 320 [arXiv:1406.2979] [INSPIRE].

[9] CMS collaboration, Measurement of jet fragmentation in $\mathrm{PbPb}$ and $\mathrm{pp}$ collisions at $\sqrt{s_{N N}}=2.76$ TeV, Phys. Rev. C 90 (2014) 024908 [arXiv:1406.0932] [InSPIRE].

[10] ALICE collaboration, Measurement of the inclusive differential jet cross section in pp collisions at $\sqrt{s}=2.76 \mathrm{TeV}$, Phys. Lett. B 722 (2013) 262 [arXiv:1301.3475] [INSPIRE].

[11] CMS collaboration, Measurement of the ratio of inclusive jet cross sections using the anti- $k_{T}$ algorithm with radius parameters $R=0.5$ and 0.7 in pp collisions at $\sqrt{s}=7$ TeV, Phys. Rev. D 90 (2014) 072006 [arXiv: 1406.0324] [INSPIRE].

[12] S. Eckweiler, Measurement of the inclusive jet cross-section in proton-proton collisions at $\sqrt{s}$ $=7$ TeV with the ATLAS detector, CERN-THESIS-2011-145.

[13] G. Soyez, A Simple description of jet cross-section ratios, Phys. Lett. B 698 (2011) 59 [arXiv:1101.2665] [INSPIRE].

[14] J.M. Butterworth, A.R. Davison, M. Rubin and G.P. Salam, Jet substructure as a new Higgs search channel at the LHC, Phys. Rev. Lett. 100 (2008) 242001 [arXiv:0802.2470] [INSPIRE].

[15] D. Krohn, J. Thaler and L.-T. Wang, Jet Trimming, JHEP 02 (2010) 084 [arXiv:0912.1342] [INSPIRE].

[16] B. Nachman, P. Nef, A. Schwartzman, M. Swiatlowski and C. Wanotayaroj, Jets from Jets: Re-clustering as a tool for large radius jet reconstruction and grooming at the LHC, JHEP 02 (2015) 075 [arXiv: 1407.2922] [InSPIRE].

[17] A. Abdesselam, E.B. Kuutmann, U. Bitenc, G. Brooijmans, J. Butterworth et al., Boosted objects: A Probe of beyond the Standard Model physics, Eur. Phys. J. C 71 (2011) 1661 [arXiv: 1012.5412] [INSPIRE].

[18] A. Altheimer, S. Arora, L. Asquith, G. Brooijmans, J. Butterworth et al., Jet Substructure at the Tevatron and LHC: New results, new tools, new benchmarks, J. Phys. G 39 (2012) 063001 [arXiv: 1201.0008] [INSPIRE].

[19] A. Altheimer, A. Arce, L. Asquith, J. Backus Mayes, E. Bergeaas Kuutmann et al., Boosted objects and jet substructure at the LHC. Report of BOOST2012, held at IFIC Valencia, 23rd-27th of July 2012, Eur. Phys. J. C 74 (2014) 2792 [arXiv:1311.2708] [INSPIRE].

[20] T. Plehn and M. Spannowsky, Top Tagging, J. Phys. G 39 (2012) 083001 [arXiv: 1112.4441] [INSPIRE].

[21] F.J. Tackmann, J.R. Walsh and S. Zuberi, Resummation Properties of Jet Vetoes at the LHC, Phys. Rev. D 86 (2012) 053011 [arXiv: 1206.4312] [InSPIRE]. 
[22] M.H. Seymour, Jet shapes in hadron collisions: Higher orders, resummation and hadronization, Nucl. Phys. B 513 (1998) 269 [hep-ph/9707338] [INSPIRE].

[23] E. Gerwick, S. Schumann, B. Gripaios and B. Webber, QCD Jet Rates with the Inclusive Generalized kt Algorithms, JHEP 04 (2013) 089 [arXiv: 1212.5235] [INSPIRE].

[24] S. Catani, M. Fontannaz, J.P. Guillet and E. Pilon, Isolating Prompt Photons with Narrow Cones, JHEP 09 (2013) 007 [arXiv: 1306.6498] [INSPIRE].

[25] S. Alioli and J.R. Walsh, Jet Veto Clustering Logarithms Beyond Leading Order, JHEP 03 (2014) 119 [arXiv:1311.5234] [INSPIRE].

[26] A. von Manteuffel, R.M. Schabinger and H.X. Zhu, The Complete Two-Loop Integrated Jet Thrust Distribution In Soft-Collinear Effective Theory, JHEP 03 (2014) 139 [arXiv: 1309.3560] [INSPIRE].

[27] S. Catani, Y.L. Dokshitzer, M. Olsson, G. Turnock and B.R. Webber, New clustering algorithm for multi - jet cross-sections in $e^{+} e^{-}$annihilation, Phys. Lett. B 269 (1991) 432 [INSPIRE].

[28] Y.L. Dokshitzer, G.D. Leder, S. Moretti and B.R. Webber, Better jet clustering algorithms, JHEP 08 (1997) 001 [hep-ph/9707323] [INSPIRE].

[29] M. Wobisch and T. Wengler, Hadronization corrections to jet cross-sections in deep inelastic scattering, hep-ph/9907280 [INSPIRE].

[30] M. Cacciari, G.P. Salam and G. Soyez, The Anti-k(t) jet clustering algorithm, JHEP 04 (2008) 063 [arXiv: 0802.1189] [INSPIRE].

[31] G.P. Salam and G. Soyez, A Practical Seedless Infrared-Safe Cone jet algorithm, JHEP 05 (2007) 086 [arXiv: 0704.0292] [INSPIRE].

[32] Y.L. Dokshitzer, V.A. Khoze, A.H. Mueller and S.I. Troian, Basics of perturbative QCD, Gif-sur-Yvette, France: Ed. Frontieres, 1991, pg. 274.

[33] R.K. Ellis, W.J. Stirling and B.R. Webber, QCD and collider physics, Camb. Monogr. Part. Phys. Nucl. Phys. Cosmol. 8 (1996) 1 [INSPIRE].

[34] H1 collaboration, A. Aktas et al., Measurement of inclusive jet production in deep-inelastic scattering at high $Q^{2}$ and determination of the strong coupling, Phys. Lett. B 653 (2007) 134 [arXiv:0706.3722] [INSPIRE].

[35] ZEUS collaboration, H. Abramowicz et al., Inclusive-jet cross sections in NC DIS at HERA and a comparison of the $k T$, anti-kT and SIScone jet algorithms, Phys. Lett. B 691 (2010) 127 [arXiv: 1003.2923] [INSPIRE].

[36] CDF collaboration, T. Aaltonen et al., Measurement of the Inclusive Jet Cross Section at

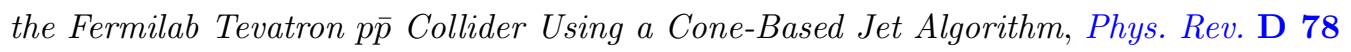
(2008) 052006 [Erratum ibid. D 79 (2009) 119902] [arXiv:0807.2204] [INSPIRE].

[37] D0 collaboration, V.M. Abazov et al., Measurement of the inclusive jet cross section in $p \bar{p}$ collisions at $\sqrt{s}=1.96 \mathrm{TeV}$, Phys. Rev. D 85 (2012) 052006 [arXiv:1110.3771] [INSPIRE].

[38] ATLAS collaboration, Measurement of the inclusive jet cross-section in proton-proton collisions at $\sqrt{s}=7 \mathrm{TeV}$ using $4.5 \mathrm{fb}^{-1}$ of data with the ATLAS detector, JHEP 02 (2015) 153 [arXiv:1410.8857] [INSPIRE].

[39] CMS collaboration, Measurements of differential jet cross sections in proton-proton collisions at $\sqrt{s}=7$ TeV with the CMS detector, Phys. Rev. D 87 (2013) 112002 [arXiv: 1212.6660] [INSPIRE]. 
[40] J. Currie, A. Gehrmann-De Ridder, E.W.N. Glover and J. Pires, NNLO QCD corrections to jet production at hadron colliders from gluon scattering, JHEP 01 (2014) 110 [arXiv: 1310.3993] [INSPIRE].

[41] R. Boughezal, F. Caola, K. Melnikov, F. Petriello and M. Schulze, Higgs boson production in association with a jet at next-to-next-to-leading order in perturbative QCD, JHEP 06 (2013) 072 [arXiv: 1302.6216] [INSPIRE].

[42] D. de Florian, P. Hinderer, A. Mukherjee, F. Ringer and W. Vogelsang, Approximate next-to-next-to-leading order corrections to hadronic jet production, Phys. Rev. Lett. 112 (2014) 082001 [arXiv:1310.7192] [INSPIRE].

[43] M. Botje, QCDNUM: Fast QCD Evolution and Convolution, Comput. Phys. Commun. 182 (2011) 490 [arXiv: 1005.1481] [INSPIRE].

[44] A. Vogt, Efficient evolution of unpolarized and polarized parton distributions with QCD-PEGASUS, Comput. Phys. Commun. 170 (2005) 65 [hep-ph/0408244] [INSPIRE].

[45] G.P. Salam and J. Rojo, A Higher Order Perturbative Parton Evolution Toolkit (HOPPET), Comput. Phys. Commun. 180 (2009) 120 [arXiv:0804.3755] [inSPIRE].

[46] V. Bertone, S. Carrazza and J. Rojo, APFEL: A PDF Evolution Library with QED corrections, Comput. Phys. Commun. 185 (2014) 1647 [arXiv:1310.1394] [INSPIRE].

[47] M. Cacciari, M. Dasgupta, F. Dreyer, G.P. Salam and G. Soyez, in preparation.

[48] ATLAS collaboration, Measurement of the correlation of jets with high $p_{T}$ isolated prompt photons in lead-lead collisions at sqrts ${ }_{N N}=2.76 \mathrm{TeV}$ with the ATLAS detector at the LHC, ATLAS-CONF-2012-121.

[49] CMS collaboration, Studies of jet quenching using isolated-photon+jet correlations in $\mathrm{PbPb}$ and pp collisions at $\sqrt{s_{N N}}=2.76 \mathrm{TeV}$, Phys. Lett. B 718 (2013) 773 [arXiv:1205.0206] [INSPIRE].

[50] A. Banfi, G.P. Salam and G. Zanderighi, $N L L+N N L O$ predictions for jet-veto efficiencies in Higgs-boson and Drell-Yan production, JHEP 06 (2012) 159 [arXiv:1203.5773] [INSPIRE].

[51] A. Banfi, P.F. Monni, G.P. Salam and G. Zanderighi, Higgs and Z-boson production with a jet veto, Phys. Rev. Lett. 109 (2012) 202001 [arXiv:1206.4998] [INSPIRE].

[52] T. Becher and M. Neubert, Factorization and NNLL Resummation for Higgs Production with a Jet Veto, JHEP 07 (2012) 108 [arXiv: 1205.3806] [inSPIRE].

[53] T. Becher, M. Neubert and L. Rothen, Factorization and $N^{3} L L_{p}+N N L O$ predictions for the Higgs cross section with a jet veto, JHEP 10 (2013) 125 [arXiv: 1307.0025] [INSPIRE].

[54] I.W. Stewart, F.J. Tackmann, J.R. Walsh and S. Zuberi, Jet $p_{T}$ resummation in Higgs production at $N N L L^{\prime}+N N L O$, Phys. Rev. D 89 (2014) 054001 [arXiv:1307.1808] [INSPIRE].

[55] A. Banfi, P.F. Monni and G. Zanderighi, Quark masses in Higgs production with a jet veto, JHEP 01 (2014) 097 [arXiv: 1308.4634] [INSPIRE].

[56] S. Catani and M.H. Seymour, A General algorithm for calculating jet cross-sections in NLO QCD, Nucl. Phys. B 485 (1997) 291 [Erratum ibid. B 510 (1998) 503] [hep-ph/9605323] [INSPIRE].

[57] A. Banfi, G.P. Salam and G. Zanderighi, Infrared safe definition of jet flavor, Eur. Phys. J. C 47 (2006) 113 [hep-ph/0601139] [INSPIRE]. 
[58] J. Gallicchio and M.D. Schwartz, Quark and Gluon Tagging at the LHC, Phys. Rev. Lett. 107 (2011) 172001 [arXiv:1106.3076] [INSPIRE].

[59] J. Gallicchio and M.D. Schwartz, Quark and Gluon Jet Substructure, JHEP 04 (2013) 090 [arXiv:1211.7038] [INSPIRE].

[60] A.J. Larkoski, G.P. Salam and J. Thaler, Energy Correlation Functions for Jet Substructure, JHEP 06 (2013) 108 [arXiv: 1305.0007] [INSPIRE].

[61] A.J. Larkoski, J. Thaler and W.J. Waalewijn, Gaining (Mutual) Information about Quark/Gluon Discrimination, JHEP 11 (2014) 129 [arXiv:1408.3122] [INSPIRE].

[62] ATLAS collaboration, Light-quark and gluon jet discrimination in pp collisions at $\sqrt{s}=7 \mathrm{TeV}$ with the ATLAS detector, Eur. Phys. J. C 74 (2014) 3023 [arXiv:1405.6583] [INSPIRE].

[63] CMS Collaboration, Performance of quark/gluon discrimination in $8 \mathrm{TeV}$ pp data, CMS-PAS-JME-13-002.

[64] M. Dasgupta and G.P. Salam, Resummation of nonglobal QCD observables, Phys. Lett. B 512 (2001) 323 [hep-ph/0104277] [INSPIRE].

[65] Y. Delenda, R. Appleby, M. Dasgupta and A. Banfi, On QCD resummation with $k(t)$ clustering, JHEP 12 (2006) 044 [hep-ph/0610242] [INSPIRE].

[66] G.P. Korchemsky and G.F. Sterman, Nonperturbative corrections in resummed cross-sections, Nucl. Phys. B 437 (1995) 415 [hep-ph/9411211] [INSPIRE].

[67] M. Dasgupta, L. Magnea and G.P. Salam, Non-perturbative QCD effects in jets at hadron colliders, JHEP 02 (2008) 055 [arXiv:0712.3014] [InSPIRE].

[68] M. Dasgupta, A. Fregoso, S. Marzani and G.P. Salam, Towards an understanding of jet substructure, JHEP 09 (2013) 029 [arXiv: 1307.0007] [INSPIRE].

[69] M. Cacciari, G.P. Salam and G. Soyez, FastJet User Manual, Eur. Phys. J. C 72 (2012) 1896 [arXiv: 1111.6097] [INSPIRE]. 